Ecole d'Eté Systèmes Optiques

\title{
Composants actifs
}

\author{
A. Carenco
}

CNET/Laboratoire de Bagneux, 196 Avenue H. Ravera, F-92220 Bagnewx, France

\section{1 - EFFETS ELECTRO-OPTIQUES}

\section{1-A-GENERALITES}

Nous allons discuter dans ce paragraphe de la propagation de la lumière à travers un milieu soumis à un champ électrique externe.

De façon générale, quand un champ électromagnétique est appliqué à un milieu, il en perturbe les propriétés diélectriques. Dans un milieu isotrope, une birefringence est induite; dans un milieu anisotrope, la birefringence est altérée.

* Si la modification d'indice de réfraction est proportionnelle au champ appliqué, on parle d'effet électrooptique linéaire ou d'effet Pockels.

* Si elle est proportionnelle au carré du champ, il s'agit alors de l'effet électro optique quadratique ou effet Kerr. Cet effet est normalement négligé quand l'effet linéaire est présent.

En effet, lorsqu'un matériau est soumis à un champ électromagnétique $\vec{E}$, il répond par une polarisation $\vec{P}$ que l'on peut écrire sous la forme d'un đéveloppement limite:

$\vec{P}=\varepsilon_{0}\left(\sum_{i} \chi^{1} \vec{E}_{i}+\sum_{(i, j)} \chi^{2} \vec{E}_{i} \vec{E}_{j}+\sum_{(i, j, k)} \chi^{3} \vec{E}_{i} \vec{E}_{j} \vec{E}_{k}+\ldots\right)$ 
où $\varepsilon_{0}$ est la constante diélectrique du vide, où les $\vec{E}_{i}$ sont les différentes composantes du champ $\vec{E}$ et les $\chi^{n}$ les tenseurs caractéristiques du matériau.

Le tenseur $\chi_{1}$ estle tenseur diélectrique qui représente la réponse linéaire usuelle (réfraction et absorption); les termes d'ordre supérieur sont les termes non linéaires qui ne deviennent importants que lorsque les champs électromagnétiques sont forts. Le terme en $\chi^{2}$ permet de rendre compte des interactions de deux champs électromagnétiques, soit optique et électrique (effet Pockels), soit optiques (interactions paramètriques à deux ondes: génération d' harmonique, somme ou différence de fréquence, amplification paramètrique,...).

\section{1-B - Effet POCKELS}

L'effet Pockels est observé uniquement dans les cristaux qui ne présentent pas de centre de symétrie cristalline. Il correspond à un changement des indices de réfraction proportionnel au champ électrique appliqué

Dans un cristal ce changement d'indice induit par le champ électrique peut être décrit par la modification de l'ellipsoïde des indices:

$\left(1 / n^{2}\right)_{1} x^{2}+\left(1 / n^{2}\right)_{2} y^{2}+\left(1 / n^{2}\right)_{3} z^{2}+2\left(1 / n^{2}\right)_{4} y z+2\left(1 / n^{2}\right)_{5} x z+2\left(1 / n^{2}\right)_{6} x y=1$

si $x, y, z$ sont choisis paralleles aux axes principaux du cristal et $\operatorname{si} n_{1}, n_{2}, n_{3}$ sont les valeurs des indices principaux associés.

La perturbation linéaire des coefficients due au champ électrique $\vec{E}$ de composantes $\mathrm{E}_{\mathrm{j}}$ se traduit par:

$\Delta\left(1 / n^{2}\right)_{i}=\sum_{j=1}^{3} r_{i j} E_{j}$

où $\mathrm{i}=1,2,3,4,5,6$ et où $\mathrm{j}=1,2,3$ sont associés à $\mathrm{x}, \mathrm{y}, \mathrm{z}$ respectivement.

Par convention est adoptée la notation contractée suivante:

$11 \rightarrow 1$

$22 \rightarrow 2$

$33 \rightarrow 3$

$23,32 \rightarrow 4$

$13,31 \rightarrow 5$

$12,21 \rightarrow 6$ 
Elle tient compte de la symétrie de permutation $r_{i j \mathrm{k}}=r_{j i \mathrm{k}}$ qui réduit le nombre de coefficients du tenseur $\left[r_{i j}\right.$ ] de 27 à 18 éléments. [ $\left.r_{i j}\right]$ de taille $6 \times 3$ est appelée le tenseur électro-optique.

L'équation peut s'écrire sous forme matricielle,

$\left(\begin{array}{c}\Delta\left(\frac{1}{n^{2}}\right)_{1} \\ \Delta\left(\frac{1}{n^{2}}\right)_{2} \\ \cdot \\ \cdot \\ \dot{\cdot}\left(\frac{1}{n^{2}}\right)_{6}\end{array}\right)=\left(\begin{array}{ccc}r_{11} & r_{12} & r_{13} \\ r_{21} & r_{22} & r_{23} \\ \cdot & \cdot & \cdot \\ \cdot & \cdot & \cdot \\ \cdot & \cdot & \cdot \\ r_{61} & r_{62} & r_{63}\end{array}\right)\left(\begin{array}{l}E_{1} \\ E_{2} \\ E_{33}\end{array}\right)$

Pour la plupart des classes cristallines non centro-symétriques, il n'existe qu'un petit nombre de coefficients électro-optiques non nuls. Comme l'indique le tableau I, ils ont pour valeur typique quelques pm/V. Par ailleurs, il apparait que l'effet électro-optique dépend des orientations du champ électrique et de la polarisation de la lumiere par rapport a la coupe du cristal. Par exemple l'usage du coefficient $\mathrm{r}_{13}$ oblige à appliquer le champ suivant l'axe $\mathrm{z}$ et à faire propager selon 1'axe y une lumière polarisée linéairement suivant $x$; le changement d'indice est alors induit selon l'axe $x$.

En général, les axes principaux de l'ellipsoïde des indices perturbé ne coincident pas avec les axes à champ nul; il s'agit donc de chercher à partir de l'équation de l'ellipsö̈de des indices les nouveaux axes et les valeurs des indices de réfraction associés. 


\begin{tabular}{|c|c|c|c|c|c|c|c|c|}
\hline CRISTAL & SYMETRIE & $\begin{array}{l}\text { TRANSMISSION } \\
\text { OPTIQUE } \\
(\mu \mathrm{m})\end{array}$ & $\begin{array}{l}\text { INDIC } \\
\text { REFR/ } \\
\text { no }\end{array}$ & $\begin{array}{l}\text { EDE } \\
\text { ICTION } \\
\text { ne }\end{array}$ & $\lambda_{(\mu \mathrm{m})}^{\lambda}$ & $\begin{array}{l}\text { COEFFICIENT } \\
\text { ELECTRO-OPTIQUE } \\
(\mathrm{pm} / \mathrm{V})\end{array}$ & $\begin{array}{l}n^{3} r_{y} \\
\max \\
(\mathrm{pm} / \mathrm{V})\end{array}$ & $\varepsilon$, \\
\hline KDP & $\overline{4} 2 m$ & $0.25-1.7$ & 1.512 & 1.470 & 0.546 & $\begin{array}{l}\mathrm{r} 41=\mathrm{r} 52=8.77 \\
\mathrm{r} 63=10.5\end{array}$ & 35 & $\begin{array}{l}45 \mathrm{tc} \\
20 / / \mathrm{c}\end{array}$ \\
\hline $\mathrm{KD} * \mathrm{P}$ & $\overline{4} 2 m$ & $0.19-2.15$ & 1.508 & 1.468 & 0.546 & $\begin{array}{l}r 41=r 52=8.8 \\
r 63=26.4\end{array}$ & 89 & $50 / / \mathrm{c}$ \\
\hline ADP & $\overline{4} 2 m$ & $0.125-1.7$ & 1.526 & 1.481 & 0.546 & $\begin{array}{l}\mathrm{r} 41=\mathrm{r} 52=24.5 \\
\mathrm{r} 63=8.5\end{array}$ & 83 & $12 / / \mathrm{c}$ \\
\hline QUARTZ & 32 & $0.12-4.5$ & 1.544 & 1.553 & 0.589 & $\begin{array}{l}\mathrm{r} 41=0.2 \\
\mathrm{r} 63=0.93\end{array}$ & 3.5 & 4.3 \\
\hline LiNbO3 & $3 \mathrm{~m}$ & $0.4-5$ & 2.297 & 2.208 & 0.633 & $\begin{array}{ll}\mathrm{r} 33=30.8 & \mathrm{r} 13=8.6 \\
\mathrm{r} 51=28 & \mathrm{r} 22=3.4\end{array}$ & 328 & $\begin{array}{l}28 / 1 \mathrm{c} \\
43 \mathrm{dc}\end{array}$ \\
\hline LiTaO3 & $3 \mathrm{~m}$ & $0.45-5$ & 2.183 & 2.188 & 0.60 & $\begin{array}{ll}\mathrm{r} 33=33 & \mathrm{r} 13=8 \\
\mathrm{r} 51=20 & \mathrm{r} 22=1 \\
\end{array}$ & 342 & 50 \\
\hline GaAs & $\overline{4} 3 m$ & $1.0-15$ & 3.42 & & 1.15 & $\mathrm{r} 41=-1.4$ & 56 & 12 \\
\hline InP & $\overline{4} 3 m$ & $1.0-12$ & 3.2 & & 1.15 & $\mathrm{r} 41=-1.6$ & 52 & 10 \\
\hline $\mathrm{ZnS}$ & $\overline{4} 3 m$ & 0,35 - & 2.364 & & 0.6 & $\mathrm{r} 4 \mathrm{l}=2,1$ & 28 & 12 \\
\hline
\end{tabular}




\section{Exemples d'effet Pockels :}

\section{LES CRISTAUX DE KDP:}

Le KDP $\left(\mathrm{KH}_{2} \mathrm{PO}_{4}\right)$ fait partie avec le KD'P et l'ADP, des cristaux électro-optiques les plus employés de part leur bonne tenue mécanique, leur transparence dans le visible et leur disponibilité en grande dimension. Le cristal est de symétrie $\overline{4} 2 m$ et son tenseur électro-optique prend la forme suivante:

$$
\begin{array}{llll}
0 & 0 & 0 \\
0 & 0 & 0 \\
0 & 0 & 0 \\
\mathrm{r}_{41} & 0 & 0 \\
0 & \mathrm{r}_{41} & 0 \\
0 & 0 & \mathrm{r}_{63}
\end{array} \mid
$$

Les cristaux sont naturellement uniaxes (indices $\mathrm{n}_{\mathrm{e}}$ et $\mathrm{n}_{\mathrm{o}}$ ). Ils deviennent biaxes en présence d'un champ électrique $\vec{E}$ de composantes $\mathrm{E}_{x}, \mathrm{E}_{\mathrm{y}}, \mathrm{E}_{2}$.

D'après ce qui précède, le nouvel ellipsoïde des indices s'écrit:

$\left(x^{2}+y^{2}\right) / n_{o}^{2}+z^{2} / n_{e}^{2}+2 r_{41} E_{x} y z+2 r_{41} E_{y} x z+2 r_{63} E_{z} x y=1$

Quand $\vec{E}$ est parallèle à $\mathrm{Oz}$, l'équation de l'ellipsoide des indices se réduit à:

$\left(x^{2}+y^{2}\right) / n_{o}^{2}+z^{2} / n_{e}^{2}+2 r_{63} E_{z} x y=1$

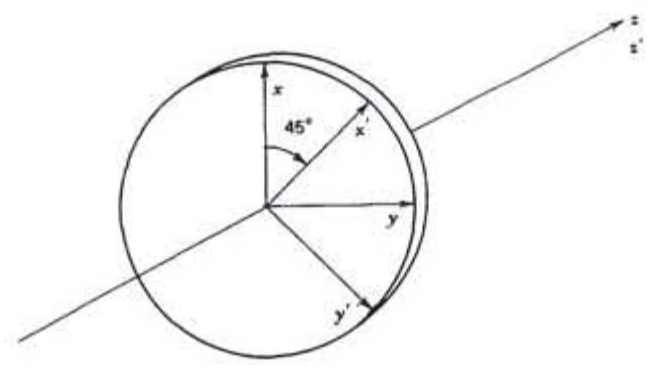

$\mathrm{Oz}$ reste l'un des axes principaux de l'ellipsoide des indices. La section par le plan $\mathrm{z}=0$ devient une ellipse dont les axes $\mathrm{Ox}^{\prime}$ et $\mathrm{Oy}^{\prime}$ sont a $45^{\circ}$ des axes cristallographiques $\mathrm{Ox}$ et $\mathrm{Oy}$. En rapportant l'équation de l'ellipsoide aux axes $\mathrm{Ox}^{\prime}, \mathrm{Oy}$ ' et $\mathrm{Oz}$, on obtient les indices principaux $\mathrm{n}_{\mathrm{x}}$, et $\mathrm{n}_{\mathrm{y}}$, relatifs à Ox' et $\mathrm{Oy}$ ': 
$1 / n_{x^{\prime}}^{2}=1 / n_{o}^{2}+r_{63} E_{z}$
$1 / n_{y^{\prime}}^{2}=1 / n_{o}^{2}-r_{63} E_{z}$

soit approximativement puisque $r_{63} E_{z} \ll n_{0}^{-2}$ :

$n_{x}=n_{e}-\Delta n$

$n_{y}=n_{o}+\Delta n$

avec $\Delta n=\frac{1}{2} n_{o}^{3} r_{63} E_{2}$,

alors que $n_{z}=n_{e}$,

En pratique, avec le champ $\vec{E}$ paralllele à l'axe optique $\mathrm{Oz}$, le cristal peut être utilisé suivant deux coupes:

* en configuration longitudinale pour laquelle le champ électrique est appliqué parallelement à la direction de propagation du faisceau,

* en configuration transverse pour laquelle le champ est appliqué perpendiculairement à la direction de propagation.

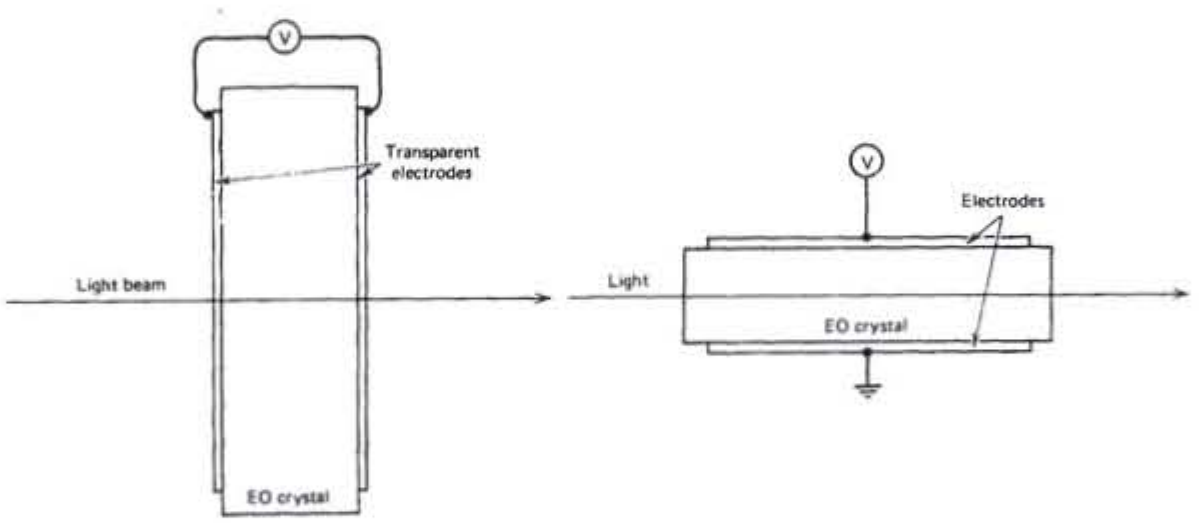

Effetelectrooptique longitudinal:

Le retard optique introduit par le champ E à la traversée du cristal de longueur $\mathrm{L}$ est proportionnel a la différence des indices $\mathrm{n}_{x^{\prime}}$ et $\mathrm{n}_{y^{\prime}}$ et vaut: 
$\Gamma=\frac{2 \pi}{\lambda} n_{o}^{3} r_{63} E_{2} L=\frac{2 \pi}{\lambda} n_{o}^{3} r_{63} V$

où $\mathrm{V}$ est la tension appliquée aux électrodes pour créer le champ électrique longitudinal.

On a donc obtenu un déphasage proportionnel à $\mathrm{V}$.

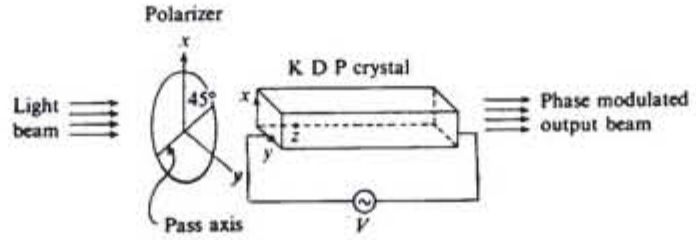

Entre polariseur et analyseur croisés, la modulation de phase induite par le champ électrique se traduit par une modulation d'intensité donnée par :

$I_{T}=I_{o} \sin ^{2} \frac{\Gamma}{2}=\sin ^{2} \frac{\pi}{2} \frac{V}{V_{\kappa}}$

On note $V_{\kappa}$ la tension qu'il faut appliquer au cristal pour obtenir une lame $1 / 2$ onde $(\Gamma=\pi)$.

$V_{\mathrm{x}}=\lambda\left(2 n_{o}^{3} r_{63}\right)$

qui est indépendant de $\mathrm{L}$.

\section{Effet électrooptique transverse}

C'est la configuration la plus utilisée. Pour une onde polarisée linéairement, le retard optique prend la forme suivante:

$\Gamma=\frac{2 \pi}{\lambda} L\left\{\left(n_{e}-n_{o}\right)-\frac{1}{2} n_{o}^{3} r_{63} E_{z}\right\}$

Il apparait une birefringence naturelle et une biréfringence électrique. La birefringence naturelle peut être compensée en choisissant une longueur $\mathrm{L}$ de cristal telle que $2 \pi / \lambda L\left(n_{e}-n_{o}\right)$ soit un multiple de $2 \pi$. Entre polariseurs croisés, l'intensité transmise s'écrit alors:

$I_{T}=I_{o} \sin ^{2}\left\{\frac{\pi}{2} \frac{V}{V_{\mathrm{r}}}\right\}$

où $\mathrm{V}=\mathrm{E}_{\mathrm{z}} \mathrm{d}$.

$V_{\mathrm{x}}=\frac{\lambda}{n_{o}^{3} r_{63}} \frac{d}{L}$ 
Cette configuration transverse fait intervenir un facteur de forme $\mathrm{d} / \mathrm{L}$ dont on peut tirer parti par rapport à la configuration longitudinale précédente. On verra plus loin comment l'optique guidée tire complètement profit de ce facteur géomètrique pour réduire considérablement la tension de commande.

\section{LES CRISTAUX DE LiNbO3 et de LiTaO3}

Ces cristaux de symétrie $3 \mathrm{~m}$ ont une grande importance, en particulier le niobate de lithium. L' effet électro-optique y est plus fort que dans KDP ou ADP. Leur tenseur électrooptique (symétrie $3 \mathrm{~m}$ ) comprend les éléments suivants:

$[\mathrm{r}]=\left|\begin{array}{ccc}0 & -r_{22} & r_{13} \\ 0 & r_{22} & r_{13} \\ 0 & 0 & r_{33} \\ 0 & r_{51} & 0 \\ r_{51} & 0 & 0 \\ -r_{22} & 0 & 0\end{array}\right|$

L'application d'un champ électrique selon $\mathrm{Oz}$ (axe optique) modifie l'ellipsoide des indices de ces cristaux uniaxes $\left(\mathrm{n}_{\mathrm{o}}\right.$ et $\left.\mathrm{n}_{\mathrm{e}}\right)$ :

$\left(x^{2}+y^{2}\right) / n_{o}^{2}+z^{2} / n_{e}^{2}+r_{13} E_{\tau} x^{2}+r_{13} E_{2} y^{2}+r_{33} E_{z} z^{2}=1$

Il apparait que les axes principaux sont inchangés et que les nouveaux indices valent:

$n_{x}=n_{o}-1 / 2 n_{o}^{3} r_{13} E_{z}$

$n_{y}=n_{o}-1 / 2 n_{o}^{3} r_{13} E_{z}$

$n_{z}=n_{e}-1 / 2 n_{e}^{3} r_{33} E_{z}$ 


\section{Configuration longitudinale}

Une lumière se propageant suivant $\mathrm{z}$ verra le même changement de phase quelle que soit sa polarisation puisque $\mathrm{n}_{\mathrm{x}}=\mathrm{n}_{\mathrm{y}}$. On peut donc faire un modulateur de phase indépendant de la polarisation de la lumière.

$V_{\mathrm{x}}=\frac{\lambda}{n_{o}^{3} r_{13}}$

Par contre on ne pourra pas induire de modulation d'amplitude.

\section{Configuration transverse}

* Si une lumière polarisée à $45^{\circ}$ de l'axe $\mathrm{z}$ dans le plan $\mathrm{xz}$ se propage selon l'axe $\mathrm{y}$, alors le retard après le parcours d'une distance $\mathrm{L}$ depuis l'entrée sera égal à :

$\Gamma=2 \pi / \lambda\left(n_{z}-n_{x}\right) L=2 \pi / \lambda\left(n_{e}-n_{o}\right) L-2 \pi / \lambda\left(n_{e}^{3} r_{33}-n_{o}^{3} r_{13}\right) / 2 \frac{L}{d} V$

où d est la distance séparant les électrodes.

On en déduit la valeur de $V_{\mathrm{n}}$ :

$V_{\pi}=\frac{\lambda}{n_{e}^{3} r_{33}-n_{o}^{3} r_{13}} \frac{d}{L}$

* Si maintenant la lumière est polarisée selon z, alors l'application d'un champ électrique également suivant $\mathrm{z}$ conduira à une modulation de phase où le déphasage sera égal à :

$\Gamma=\frac{2 \pi}{\lambda} n_{e} L-\frac{\pi}{\lambda} n_{e}^{3} r_{33} \frac{L}{d} V$

et $V_{\kappa}=\frac{\lambda}{n_{e} r_{33}} \frac{d}{L}$

ce qui est plus efficace que dans la situation précédente puisque $r_{33}$ et $r_{13}$ ont le même signe.

\section{1-C - EEFET KERR}

L'effet Kerr à la différence de l'effet Pockels existe quelle que soit la symétrie du milieu. L'équation de l'ellipsoïde des indices s'écrit: 
$x^{2}\left\{\frac{1}{n_{1}^{2}}+s_{11} E_{x}^{2}+s_{12} E_{y}^{2}+\ldots+2 s_{16} E_{x} E_{y}\right\}+y^{2}\left\{\frac{1}{n_{2}^{2}}+s_{21} E_{x}^{2}+\ldots+2 s_{20} E_{x} E_{y}\right\}+z^{2}\left\{\frac{1}{n_{3}^{2}}+s_{31} E_{x}^{2}+\ldots+2 s_{36} E_{x} E_{y}\right\}$

$+2 y z\left\{s_{41} E_{x}^{2}+. .+2 s_{46} E_{x} E_{y}\right\}+2 z x\left\{s_{51} E_{x}^{2}+. .+2 s_{56} E_{x} E_{y}\right\}+2 x y\left\{s_{61} E_{x}^{2}+. .+2 s_{66} E_{x} E_{y}\right\}=1$

où les éléments $s_{i j}$ de la matrice $6 \times 6$ sont les coefficients Kerr dans les axes principaux du milieu. On peut montrer que la birefringence induite est de la forme:

$\Delta n=\frac{1}{2} n^{3} s_{i j} E^{2}$

que l'on écrit:

$\Delta n=K \lambda E^{2}$

Kest appele la constante de Kerr:

$s_{\theta}=-\frac{K \lambda}{n^{3}}$

Le tableau II donne les valeurs des coefficients Kerr de quelques matériaux.

TABLEAU II: Constantes Kerr de quelques matériaux

\begin{tabular}{|c|c|c|}
\hline LIQUIDES & $\begin{array}{l}\lambda \\
(\mu \mathrm{m})\end{array}$ & $\mathrm{K}\left(\mathrm{x} 10-14 \mathrm{~m} / \mathrm{V}^{2}\right)$ \\
\hline Eau & 0.59 & 5.1 \\
\hline Sulfure de carbone & 0.63 & 3.2 \\
\hline Nitrotoluène & 0.59 & 137 \\
\hline Nitrobenzène & 0.59 & 244 \\
\hline $\mathrm{BaTiO}_{3}$ & 0.63 & 0.22 \\
\hline PLZT & 0.55 & 2.6 \\
\hline KDP & 0.55 & $3.1 \times 10^{-3}$ \\
\hline
\end{tabular}


L'effet électro-optique quadratique est observé dans la plupart des liquides (eau, $\mathrm{CS}_{2}, .$. ). La biréfringence qui est créé s'explique par l'orientation des molécules sous l'effet du champ électrique appliqué $\mathrm{E}$.

Une cellule de Kerr est constituée d'un condensateur immergé dans le liquide. Les tensions demi-onde typiques de ces cellules sont de quelques $10 \mathrm{kV} / \mathrm{cm}$. Comme les constantes de temps d'établissement des phénomènes sont extrêmement courtes $(<\mathrm{ps})$, les limitations de bande passante proviennent de l'électronique de commande. C'est la raison pour laquelle les cellules de Pockels sont préférées à ces cellules liquides.

Parmi les matériaux solides, les céramiques PLZT (matériaux polycristallins composés de $\mathrm{Pb}, \mathrm{La}, \mathrm{Zr}, \mathrm{Ti}$ ) présentent un effet Kerr important ainsi qu'une grande souplese d'utilisation, car la biréfringence induite par le champ électrique appliqué peut être ajusté par la composition du polycristal: $\mathrm{Pb} 1-\mathrm{x} \mathrm{La} \times \mathrm{Zr} 0,65$ Ti 0,35.

Ces composés sont transparents de 0,4 à $5 \mu \mathrm{m}$. En outre leur tension d'ouverture est relativement faible, de l'ordre de $100 \mathrm{a} 200 \mathrm{~V}$ pour une bande passante de quelques $\mathrm{MHz}$. Ils trouvent des applications intéressantes comme obturateurs, notamment de faisceaux de grande dimension (10 à $15 \mathrm{~cm}$ de diamètre).

Caractéristiques d'un obturateur commercial:

* Diamètre de la céramique $=50 \mathrm{~mm}$

* Tension de commande $=500 \mathrm{~V}$

* Transmission état ouvert $=30 \%$

* Transmission état fermé $<10^{-2}$

\section{1-D - ELECTRO-ABSORPTION DANS LES MATERIAUX SEMI-CONDUCTEURS}

\section{Effet FRANZ KELDYSH}

En présence d'un fort champ électrique, le bord d'absorption d'un matériau semiconducteurs est déplacé vers les grandes longueurs d'onde comme illustré sur la figure dans le cas de GaAs soumis à un champ de $1,3 \times 10^{5} \mathrm{~V} / \mathrm{cm}$. Dans les matériaux à bande interdite directe comme les semiconducteurs III V ( $\mathrm{GaAs}$, InP..) le bord de bande étant très abrupt,de grandes variations de l'absorption peuvent être ainsi crées; à la longueur d'onde de $9000 \mathrm{~A}^{\circ}$ le coefficient d'absorption passe de $25 \mathrm{~cm}^{-1} \mathrm{a} 10^{4} \mathrm{~cm}^{-1}$ quand le champ électrique est appliqué. 


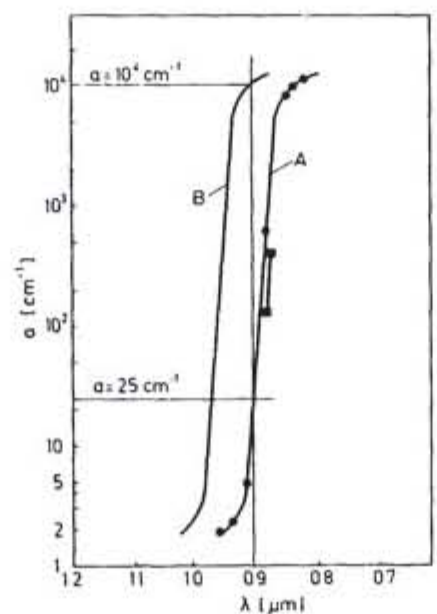

Franz-Keldysh shift of the absorption edge of GaAs. Curve $A$ is the zero field absorption curve for GaAs. (Circular dots represent experimental data points for n-type material with carrier concentratio $n=3 \times 10^{16} \mathrm{~cm}^{-3}$. For the square dots $n$ $=5.3 \times 10^{16} \mathrm{~cm}^{-3}$. Curve $B$ shows the shirted absorption edge for a field of 1.3 $\times 10^{3} \mathrm{~V} / \mathrm{cm}$

Un modulateur Franz-Keldysh typique est constitué d'une diode Schottky ou d'une jonction pn que l'on polarise en inverse afin de créer un champ électrique intense dans la zone de charge d'espace. Ces dispositifs peuvent être utilisés avec propagation de la lumière perpendiculairement au substrat (quand il est transparent), mais ils deviennent particulièrement efficaces dans le cas des ondes guidées dans le plan des couches.

La longueur du modulateur est choisie à partir de courbes telles que celles de la figure afin d'établir le compromis entre perte d'insertion mimimum au repos et profondeur de modulation maximum à la longueur d'onde de travail.

\section{1-E-APPLICATIONS:MODULATEURS}

L'effet électro optique trouve un grand domaine d'applications: modulation de phase ou d'amplitude de la lumière, Q-switching ou mode-locking des lasers,... 


\section{1 - E - a - Modulateur de phase ou d'amplitude}

Nous avons vu à travers l'étude de l'effet Pockels que l'application d'un champ électrique sur un cristal se traduisant par des variations de phase peut être exploitée pour moduler directement en phase la lumière.

La polarisation incidente est rendue parallèle à l'une des directions principales du cristal. Le champ électrique appliqué module linéairement la phase de l'onde incidente traversant le cristal. On obtient en sortie du cristal un spectre d'ondes comportant les composantes spectrales $\omega_{0} \pm n \Omega$, $\omega_{0}$ étant la frequence du laser et $\Omega$ la fréquence de modulation. L'amplitude de 1 'onde à la fréquence $\omega_{0} \pm n \Omega$ est proportionnelle à la fonction de Bessel d'ordre $\mathrm{n}: J_{n}(2 \pi / \lambda L \Delta n)$.

Dans certaines configurations, le champ électrique agit différemment sur les deux polarisations linéaires passant à travers le cristal et de ce fait il peut induire un retard entre les deux polarisations qui dépend du champ électrique. Ceci permet de moduler l'amplitude de la lumière.

\section{KDPen mode longitudinal:}

On a vu qu' une onde polarisée linéairement selon $x$ ' se propageant suivant $\mathrm{z}$ dans un cristal soumis à un champ parallèlement à z par l'intermédiaire d'électrodes polarisées à la tension $\mathrm{V}$, subissait une modulation de phase.

Si maintenant la lumière est polarisée selon y, on peut la décomposer en deux ondes de polarisation linéaire selon $x^{\prime}$ 'et $y$ ' ayant même amplitude et même phase. La traversée du cristal les déphase l'une par rapport a l'autre d'une valeur proportionnelle à V, mais indépendante de la longueur:

$\Gamma=\frac{2 \pi}{\lambda} n_{o}^{3} r_{63} V$

Quand ce déphasage relatif est égal à $\pi,\left(V=V_{k}\right)$, l'onde sort avec un état de polarisation linéaire. Sil'on fait passer cette onde à travers un analyseur par exemple croisé avec le polariseur d'entrée, alors l'amplitude de l'onde sera modulée.

$I_{T}=I_{O} \sin ^{2} \frac{\Gamma}{2}=\sin ^{2} \frac{\pi}{2} \frac{V}{V_{\pi}}$

avec $V_{\mathrm{x}}=\lambda\left(2 n_{o}^{3} r_{63}\right)$

Vers $0,6 \mu \mathrm{m}, V_{\mathrm{k}}$ vaut environ $10 \mathrm{kV}$. 


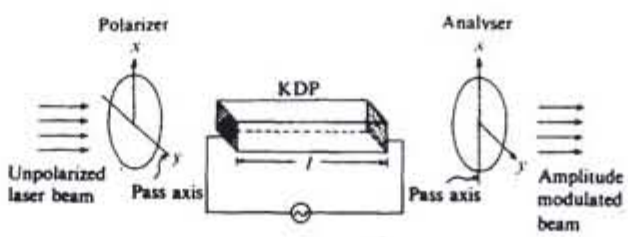

\section{KDPen mode transverse :}

Il n'y a plus besoin d'électrodes transparentes comme dans la configuration longitudinale; le retard est cette fois fonction du rapport de la largeur sur la longueur du cristal. Ceci permet de réduire la tension demi-onde.

Considérons la propagation selon $\mathrm{y}^{\prime} \mathrm{d}^{\prime}$ une onde polarisée linéairement à $45^{\circ}$ de $\mathrm{x}^{\prime}$ dans un cristal où le champ est appliqué selon z. II apparait un déphasage constant lié à la biréfringence intrinsèque du cristal ( que l'on compense avec un Babinet-Soleil par exemple) et un déphasage $\Gamma$ proportionnel a V:

$\Gamma=\frac{\pi}{\lambda} n_{o}^{3} r_{63} \frac{L}{d} V$

$V_{\mathrm{k}}=\frac{\lambda}{n_{o}^{3} r_{63}}\left(\frac{d}{L}\right)$

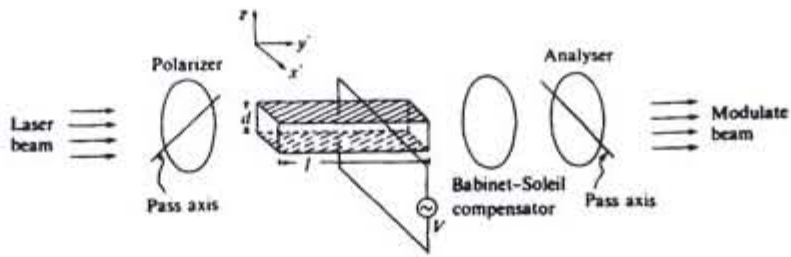

Un tel dispositif en configuration transverse requiert une tension de commande moindre. exemple : KD'P a $0,6 \mu \mathrm{m}$ avec un rapport $\mathrm{d} / \mathrm{L}$ de 0,1 a un $V_{\mathrm{x}}<700 \mathrm{~V}$.

LiNbO3 en mode transverse : 
Si la polarisation linéaire de l'onde se propageant suivant y est parallele à $\mathrm{z}$ ainsi que le champ électrique, on peut alors obtenir de la modulation de phase qui se superpose à un déphasage intrinsèque au cristal.

$V_{\mathrm{\kappa}}=\frac{\lambda}{n_{e}^{3} r_{33}} \frac{d}{L}$

qui est inférieure a $50 \mathrm{~V}$ avec $\mathrm{d}=0,25 \mathrm{~mm}$ et $\mathrm{L}=9,5 \mathrm{~mm}$ a $0,6 \mu \mathrm{m}$.

\section{1 - E - b - Considérations sur la conception d'un modulateur}

\section{Choix de la configuration:}

Il faut noter que dans la configuration longitudinale la tension de commande est independante de la longueur du cristal; la configuration transverse permet d'abaisser de façon importante cette tension,

$V_{\pi}^{\text {TRINSVERSE }}=\frac{d}{L} V_{\pi}^{\text {LONGTIUDINAL }}$

mais il est nécessaire de compenser la birefringence intinsèque au cristal en fonction de la température.

\section{Facteur géométrique:}

En configuration transverse, la tension demi-onde est proportionnelle à $d / L$. En pratique $\mathrm{d}$ et $\mathrm{L}$ ne sont pas indépendants. Sauf avec un faisceau optique guidée, la valeur minimale permise pour d est déterminée par la diffraction.

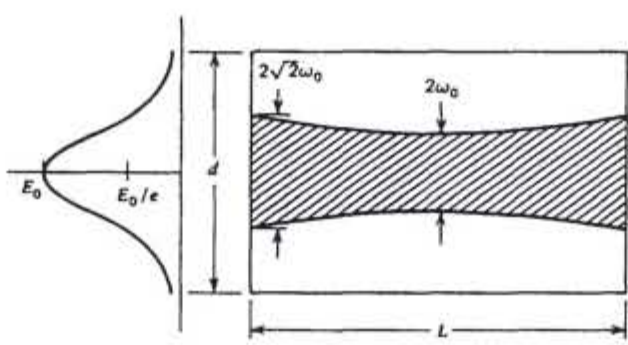

$\mathrm{Si}$ on considere un faisceau gaussien de rayon $\mathrm{w}_{0}$, on peut montrer que:

$d_{\text {optimum }}=2 S \sqrt{\frac{\lambda}{n} \frac{L}{\pi}}$ 
avec $\mathrm{S}=$ coefficient de sécurité compris typiquement entre 3 et 6 .

Exemple: $\mathrm{LiTaO}_{3}: \mathrm{L}=9,5 \mathrm{~mm}, \mathrm{n}_{\mathrm{o}} \sim \mathrm{n}_{\mathrm{e}}=2,19$

a $0,6 \mu \mathrm{m}, \mathrm{d}_{\min }=60 \mu \mathrm{m}$

pour $S=4, d=0,25 \mathrm{~mm}$

On en déduit que $V_{\mathrm{s}}=70 \mathrm{~V}$ en configuration de modulation d'amplitude et $50 \mathrm{~V}$ en modulation de phase.

\section{Aspect électrique}

Le modulateur peut se représenter électriquement par une capacité, la résistance série et la self étant a priori négligeables. Sa bande passante est donc limitée par la constante de temps RC, où R est l'impédance de la charge d'adaptation au circuit de commande que l'on place en parallele sur les électrodes du modulateur et qui vaut généralement $50 \Omega$.

On définit la puissance de commande par unité de bande passante électrique: $P / \Delta$ fen $m W / M H z$. La puissance étant dissipée en majeure partie sur la charge $\mathrm{R}$ d'adaptation:

$P=\frac{V_{m}^{2}}{2 R}$

$\Delta f=1 /(2 \pi R C)$

$P / \Delta f=\pi C V_{m}^{2}$

Avec la structure transverse, on peut calculer que:

$(P / \Delta f)_{K D P}=24 S^{2} \mathrm{~mW} / \mathrm{MHz}$

$\left(P / \Delta f_{L^{\prime N B O} O_{3}}=0,54 S^{2} \mathrm{~mW} / \mathrm{MHz}\right.$

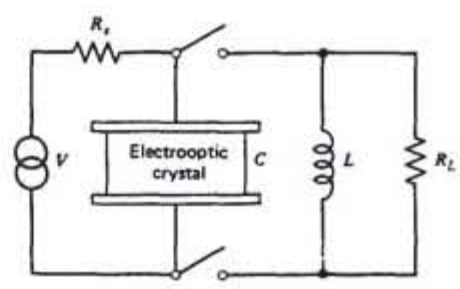

ce qui montre la supériorite du $\mathrm{LiNbO}_{3}$ par rapport au KDP. Mais le seuil de dommage est plus faible et il présente des effets photoréfractifs dans le visible.

L'introduction d'une self $L$ dans le circuit de commande du modulateur peut en faire un circuit résonant autour de la fréquence $\frac{1}{\sqrt{L C}}$ et ainsi diminuer la consommation du dispositif.

\section{Facteur de mérite}

De façon générale on a vu que la tension demi-onde dépendait du terme $n^{3} r_{i j}$; c'est le facteur de mérite qui est le plus souvent employé pour classer les matériaux présentant de l'effet 
Pockels (cf Tableau I). Il ne faut cependant pas perdre de vue que la constante diélectrique du

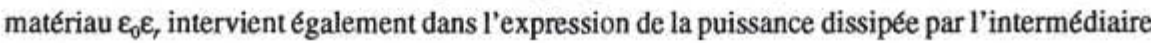
de C.

Limitation de la bande passante par le temps de transit de l'onde optique:

Pour des fonctionnements à haute fréquence, le champ électrique peur varier de façon significative pendant le temps $\tau=n L / c$ mis par la lumière pour traverser le cristal. Cette limitation peut être largement levée avec les modulateurs à ondes progressives: dans ces derniers l'onde hyperfréquence et l'onde optique se propagent codirectivement à l'intérieur du cristal. Les électrodes doivent alors constituer une ligne adaptée. L'onde optique verra donc toujours la même valeur du champ électrique (aux pertes de propagation près) si les ondes se propagent à la même vitesse. La limitation ultime résulte du désaccord de phase possible entre les deux ondes:

$f_{\max }=\frac{c}{2 L\left(n-n_{\text {microonde }}\right)}$

où les $\mathrm{n}$ désignent les indices des ondes optique et microonde.

\section{1 - E - c - Caractéristiques importantes d'un modulateur}

* Profondeur de modulation :

- dans le cas d'un mod d'amplitude, $\eta=|I(0)-I(V)| I I(0)$

- dans le cas d'un mod de phase on peut prendre la même définition en se ramenant à une modulation d'intensité; alors $\eta=\sin ^{2}(\Gamma / 2)$

\section{* Diaphotie ou taux d'extinction optique}

s'exprime en dB par: $=10 \log \left(I_{\text {TRNSSMIS }}^{\mathrm{MIN}} / I_{\text {TRANSMIS }}^{\mathrm{MNX}}\right)$

* Bande passante:

Quand elle est définie comme la différence de fréquence pour laquelle la profondeur de modulation chute de moitié, on parle de bande à $3 \mathrm{~dB}$ optique. Mais on définit souvent la bande passante a $3 \mathrm{~dB}$ électrique (profondeur de modulation réduite à $71 \%$ de sa valeur). 
Dans le cas d'un commutateur on définit usuellement le temps de commutation qui est relié à la bande passante par l'expression $T=2 \pi / \Delta f$.

\section{*Perte d'insertion}

donnée habituellement en decibels $(\mathrm{dB}) \cdot L_{i}=10 \log _{10}\left(I_{\text {Transmis }} / I_{\text {Incident }}\right)$

\section{2 - EFFETS ACOUSTO-OPTIQUES}

\section{2-A-GENERALITES}

Les contraintes mécaniques produites par une onde acoustique se propageant dans un milieu génèrent du fait de l'effet photoélastique une modification de l'indice de réfraction. Une onde acoustique variant périodiquement va produire un réseau d'indice de réfraction de même période. Une onde optique interagissant avec ce réseau va être diffractée

* soit suivant plusieurs ordres, c'est le régime Raman-Nath,

* soit suivant un seul ordre, c'est le régime de Bragg.

Ces interactions acousto-optiques ont une grande importance pratique; elles sont à la base de nombreux dispositifs de modulation ou de déflection utilisés pour l'héterodynage, l'analyse de spectre, le Q-switching ou le mode-locking des lasers.

Ce phénomène peut être décrit à partir du tenseur photoélastique du matériau. De même qu'avec l'effet électro-optique, l'effet d'une contrainte sur les propriétés optiques d'un milieu (solide ou liquide) va se traduire par une modification de l'ellipsoïde des indices. 


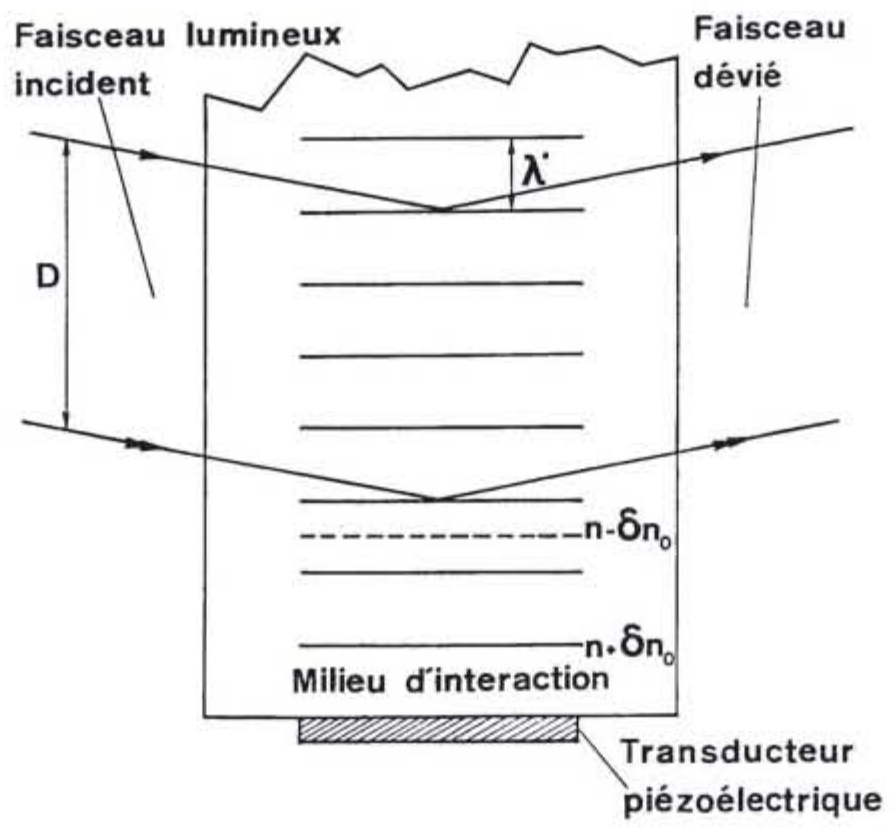

Si l'ellipsoïde des indices est rapporté dans ces axes principaux $(x, y, z)$, il peut s'écrire:

$\frac{x^{2}}{n_{1}^{2}}+\frac{y^{2}}{n_{2}^{2}}+\frac{z^{2}}{n_{3}^{2}}=1$

où $\mathrm{n}_{1}, \mathrm{n}_{2}, \mathrm{n}_{3}$ sont les indices principaux de réfraction.

En employant la notation contractée qui résulte des symétries de permutation et qui permet de simplifier l'écriture des tenseurs, $c$ 'est par le tenseur photoélastique $\left[\mathrm{p}_{i j}\right]$ qu'on relie la contrainte $\mathrm{S}$ et l'indice de refraction:

$\Delta\left(\frac{1}{n^{2}}\right)_{i}=p_{i j} S_{j}$,

$i, j=1,2, \ldots, 6$

où les $\mathrm{S}_{\mathrm{j}}$ sont les composantes de la contrainte.

$S_{1}=S_{x x}$

$S_{2}=S_{y y}$

$S_{3}=S_{z z}$

$S_{4}=S_{y z}$

$S_{5}=S_{2 x}$

$S_{6}=S_{x y}$ 
L'équation de l'ellipsoïde des indices devient donc:

$x^{2}\left\{\frac{1}{n_{1}^{2}}+\sum_{j=1}^{6} p_{1 j} S_{j}\right\}+y^{2}\left\{\frac{1}{n_{2}^{2}}+\sum_{j=1}^{6} p_{3 j} S_{j}\right\}+z^{2}\left\{\frac{1}{n_{3}^{2}}+\sum_{j=1}^{6} p_{3 j} S_{j}\right\}+2 y z\left\{\sum_{j=1}^{6} p_{4 j} S_{j}\right\}+2 z x\left\{\sum_{j=1}^{6} p_{5 j} S_{j}\right\}+2 x y\left\{\sum_{j=1}^{6} p_{6 j} S_{j}\right\}=1$

Les contraintes de façon générale modifient les dimensions et la forme de l'ellipsoïde des indices. Ce sont les symétries qui dictent les relations entre les 36 coefficients du tenseur $\mathrm{p}_{\mathrm{ij}}$.

Dans les solides monocristallins les interactions acousto-optiques dépendent donc de l'orientation par le fait de [p].

Par exemple pour un milieu isotrope (eau) $[\mathrm{p}]$ ne possède que 2 coefficients indépendants $\mathrm{p}_{11}$ et $p_{12}(=0,31$ a $0,6 \mu \mathrm{m})$.

Considérons la propagation d'une onde acoustique longitudinale suivant l'axe $\mathrm{z}$. le champ de contraintes associé à cette onde sonore est donc:

$S_{3}=S \sin (\Omega t-K z)$

où $\mathrm{K}$ est le nombre d'onde.

L'ellipsoïde des indices s'écrit:

$x^{2}\left\{\frac{1}{n^{2}}+p_{12} S \sin (\Omega t-K z)\right\}+y^{2}\left\{\frac{1}{n^{2}}+p_{12} S \sin (\Omega t-K z)\right\}+z^{2}\left\{\frac{1}{n^{2}}+p_{11} S \sin (\Omega t-K z)\right\}=1$

où $\mathrm{n}$ est l'indice de réfraction de l'eau.

Puisqu'il n'y a pas de termes croisés les axes principaux restent inchangés et les nouveaux indices de réfraction sont donnés par:

$n_{x}=n-\frac{1}{2} n^{3} p_{12} S \sin (\Omega t-K z)$

$n_{y}=n-\frac{1}{2} n^{3} p_{12} S \sin (\Omega t-K z)$

$n_{z}=n-\frac{1}{2} n^{3} p_{11} S \sin (\Omega t-K z)$

On peut noter qu'en présence de l'onde sonore l'eau devient un milieu périodique qui est équivalent à un réseau de volume avec une période de réseau égale à $2 \pi / K=\Lambda$.

Dans le cas du $\mathrm{LiNbO}_{3}$ du $\mathrm{LiTaO}_{3}$ ou du quartz qui appartiennent à la classe cristaline $3 \mathrm{~m}$, le tenseur possède 8 coefficients photoélastiques indépendants. 
On montrerait que de même une onde de cisaillement produit un réseau de volume.

TABLEAU III: Propriétés photoélastiques de quelques matériaux

\begin{tabular}{|l|l|l|l|l|}
\hline MATERIAU & $\begin{array}{l}\text { VITESSE } \\
\text { ACOUSTIQUE } \\
(\mathrm{km} / \mathrm{s})\end{array}$ & $\mathrm{M}_{1}$ & $\begin{array}{l}\text { FIGURE DE MERTTE } \\
\mathrm{M}_{2}\left(\times 1^{-15} \mathrm{~s}^{3} / \mathrm{kg}\right)\end{array}$ & $\mathrm{M}_{3}$ \\
\hline Silice & 6 & 8 & 1.5 & 1.3 \\
\hline $\mathrm{H} 2 \mathrm{O}$ & 1,5 & 4.4 & 160 & 30 \\
\hline $\mathrm{TeO} 2$ & 0,617 & 323 & 793 & 14 \\
\hline $\mathrm{PbMoO} 4$ & 3,75 & 15 & 24 & 28 \\
\hline $\mathrm{LiNbO} 3$ & 8.6 & 67 & 7 & 10 \\
\hline $\mathrm{KDP}$ & 5.5 & 9 & 4 & 1.5 \\
\hline $\mathrm{GaAs}$ & 5.1 & 925 & 104 & 180 \\
\hline
\end{tabular}

\section{2-B - CONCEPT DE BASE DES INTERACTIONS AO}

Une onde acoustique qui varie périodiquement produit donc un réseau d'indice de réfraction de période égale à la longueur d'onde acoustique. Les variations d'indice de réfraction autour de la valeur moyenne sont de l'ordre de $10^{-4}$. Une onde optique interagissant avec ce réseau va être diffractée

A partir de la valeur du changement de l'indice, que l'on peut écrire:

$\Delta n=\sqrt{M_{2} 10^{7} P_{a} / 2 A}$

, on définit le facteur de mérite de diffraction:

$M_{2}=\frac{n^{6} p^{2}}{\rho v_{a}^{3}}$

où

n est l'indice de réfraction du milieu non contraint

p est l'élément convenable du tenseur photoélastique

$P$ a est la puissance acoustique totale en $\mathrm{W}$

$\rho$ est la masse volumique 
$v_{\text {a }}$ est la vitesse sonore dans le milieu

A est la section de l'onde sonore.

Toutes les quantités sont en unités cgs, sauf $\mathrm{P}_{\mathrm{a}}$ (en watts )

L'effet est relativement faible même pour un choix optimum de matériau et d'orientation. Par exemple, a $0,63 \mu \mathrm{m} \mathrm{M}_{2}$ vaut $1.51 \times 10^{-18} \mathrm{~s}^{3} / \mathrm{gm}$ pour la silice fondue et $6,9 \times 10^{-18}$ pour $\mathrm{LiNbO}_{3}$. Pour une densité de puissance acoustique de $100 \mathrm{~W} / \mathrm{cm} 2, \Delta n$ ne dépasse pas 10-4.

Cette pertubation varie avec le temps et l'espace. S'il s'agit d'une onde progressive la perturbation va se déplacer dans le milieu à la vitesse du son $v_{\mathrm{a}}$ (typiquement quelques $10^{3} \mathrm{~m} / \mathrm{s}$ ) c'est à dire à une vitesse de quelques 5 ordres de grandeur plus faible que celle de la lumière. Ceci veut dire que la perturbation est quasi stationnaire.

Ilen résulte qu'une onde optique de vecteur d'onde $k=2 \pi n \lambda$ interagissant avec une onde sonore de vecteur d'onde $K\left(K=\frac{2 \pi}{\Lambda}\right)$ sera efficacement réfléchie dans les directions définies par

$2 k \sin \theta=m K=m \frac{2 \pi}{\Lambda}$

où $\mathrm{m}$ est un entier.

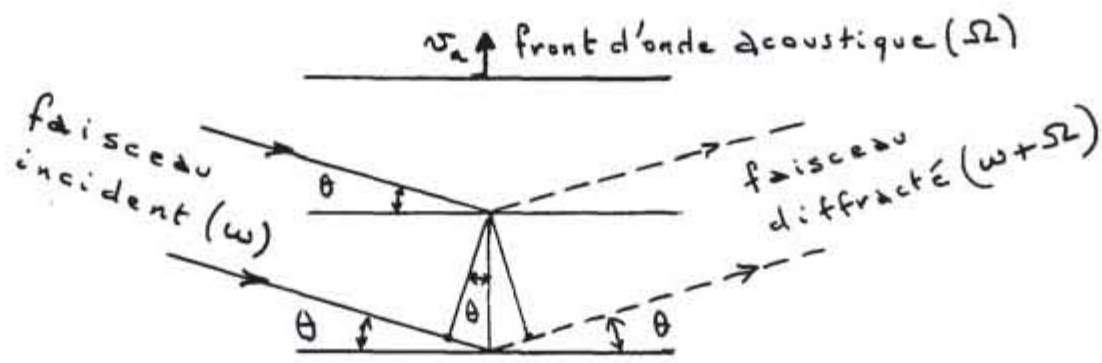

D'après le principe de conservation de l'énergie et des moments, on peut montrer que la diffraction sur plusieurs ordres (Figure a) ne peut se produire que si le milieu a une épaisseur très petite par rapport à $\Lambda$, (condition de réseau mince du régime de Raman-Nath) et que dans le cas contraire du réseau épais, cette diffraction (Figure b) se réduit à un seul ordre (cas du régime de Bragg).

Dans ce dernier cas, la relation précédente se réduit à: 


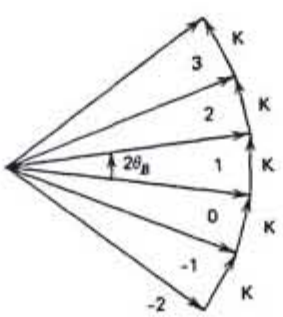

(a)
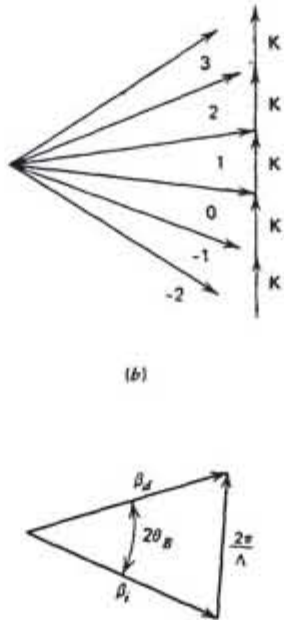

$2 k \sin \theta=K$

ou encore

$$
2 \Lambda \sin \theta=\frac{\lambda}{n}
$$

C'est la condition de Bragg.

Par ailleurs on montre que l'onde réfléchie est déplacée en fréquence de $\pm \Omega$.

On utilise le paramètre $Q$ :

$Q=\frac{2 \pi \lambda L}{n \Lambda^{2}}$

pour distinguer les deux types fondamentaux d'interaction acousto-optique.

* Si $Q \ll 1$, ou encore si $L \ll \frac{\Lambda^{2} n}{22 a}$, l'onde incidente va diffracter suivant plusieurs ordres. C'est le régime de Raman-Nath correspondant à la diffraction par un réseau mince.

* Si Q $\gg 1$, ou encore si $L \gg \frac{\Lambda^{2} n}{2 \pi \lambda}$, l'onde incidente va diffracter suivant un seul ordre. C'est le régime de Bragg correspondant à la diffraction par un réseau épais.

La tranche intermédiaire correspondant à un régime composite n'a en pratique aucun interêt. Dans tous les cas, les ondes diffractées ont une fréquence différente de la fréquence de l'onde incidente. 


\section{2 - B - 1 - REGIME RAMAN- NATH}

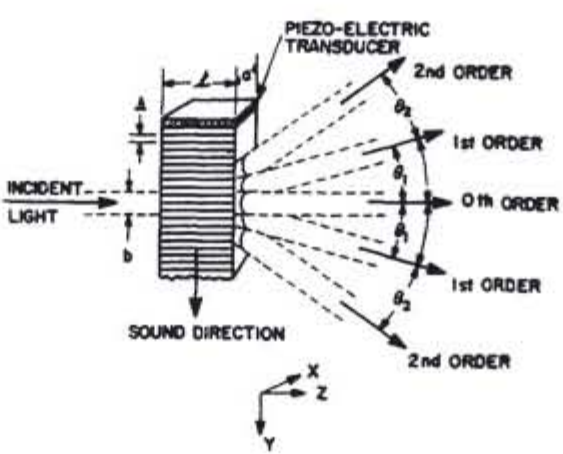

La structure de base de l'interaction est illustrée sur la figure .La lumière passant à travers le milieu dans la direction $\mathrm{z}$ subit un đéphasage donné par:

$\Delta \phi=\frac{\Delta n 2 \pi n L}{\lambda} \sin \frac{2 \pi y}{\Lambda}$

où $\Delta n$ est le changement d'indice induit par l'onde acoustique, $L$ la longueur d'interaction et $\Lambda$ la longueur d'onde acoustique.

On en déduit que :

$\Delta \phi=\frac{2 \pi n}{\lambda} \sqrt{M_{2} 10^{7} P_{s} L /(2 a)} \sin \left(\frac{2 \pi y}{\Lambda}\right)$

où a est l'épaisseur du faisceau acoustique.

La lumière incidente est alors diffractée dans plusieurs ordres suivant des angles donnés par la relation:

$\sin \theta=\frac{m \lambda}{n \Lambda} \quad m=0, \pm 1, \pm 2, \ldots$

L'intensité des différents ordres diffractés est donnée par:

$$
\begin{array}{rr}
I I I_{0}=\left\{J_{m}\left(\Delta \phi^{\prime}\right)\right\}^{2} / 2 & |m|>0 \\
\left\{J_{0}\left(\Delta \phi^{\prime}\right)\right\}^{2} & m=0
\end{array}
$$

où $\mathrm{J}$ sont les fonctions de Bessel, $\mathrm{I}_{0}$ est l'intensité optique transmise en l'absence d'onde acoustique et $\Delta \phi^{\prime}$ est la valeur maximum de $\Delta \phi$. 
Du fait de la diffraction dans plusieurs ordres et d'un indice de modulation plus faible, le régime de Bragg est plus employé.

\section{2 - B - 2 - REGIME BRAGG}

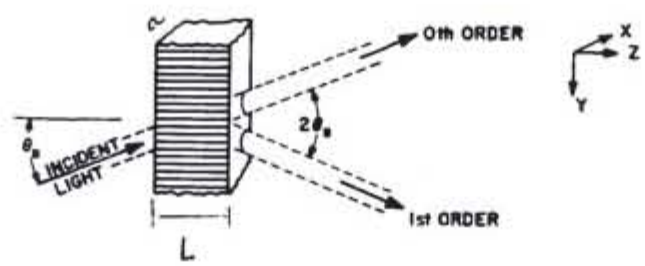

Le faisceau incident doit avoir un angle remplissant la condition de Bragg:

$\sin \theta_{B}=\frac{\lambda}{2 \Lambda n}$

Le faisceau diffracté (ordre 1) émerge alors avec un angle de $2 \theta_{B}$ par rapport au faisceau non diffacté (ordre 0) avec une intensité $\mathrm{I}_{\mathrm{D}}$ égale à: .

$\frac{I_{D}}{I_{0}}=\sin ^{2}\left(\frac{\Delta \phi^{\prime}}{2}\right)$

où $\mathrm{I}_{0}$ estl' intensité transmise en l'absence de champ acoustique. Le taux de modulation maximum vaut:

$\frac{I_{D}}{I_{0}}=\sin ^{2}\left(\frac{\pi n}{\lambda}\right) \sqrt{10^{7} M_{2} P_{a} L /(2 a)}$

\section{2-C - APPLICATIONS: MODULATEUR, DEFLECTEUR, ANALYSEUR DE SPEC-} TRE

Les principales applications sont les modulateurs de lumière, les déviateurs de lumière, les modulateurs multifréquences, les composants de traitement de signal (analyse de Fourier, convolution, filtrage,correlation, autocorrélation,...).

A côté du facteur de mérite $\mathrm{M}_{2}$, est souvent employé pour qualifier les matériaux destinés à la modulation ou à la déflexion le facteur $\mathrm{M}_{1}$. 
FACTEURS DE MERITE ACOUSTOOPTIQUE

VALEURS ET SIGNIFICATIONS

\begin{tabular}{|c|c|c|}
\hline $\begin{array}{l}\text { Facteur de } \\
\text { mérite }\end{array}$ & Valeurs & Signification $^{\dagger}$ \\
\hline$M_{1}$ & $\frac{n^{7} p^{2}}{p v}$ & $=n \Delta f_{B}$ \\
\hline$M_{2}$ & $\frac{n^{6} p^{2}}{\rho v^{3}}$ & $=n$ \\
\hline$M_{3}$ & $\frac{n^{7} p^{2}}{\rho v^{2}}$ & $=n \Delta f_{B} \tau$ \\
\hline
\end{tabular}

$M_{1}=\frac{n^{7} p^{2}}{\rho v_{a}}$

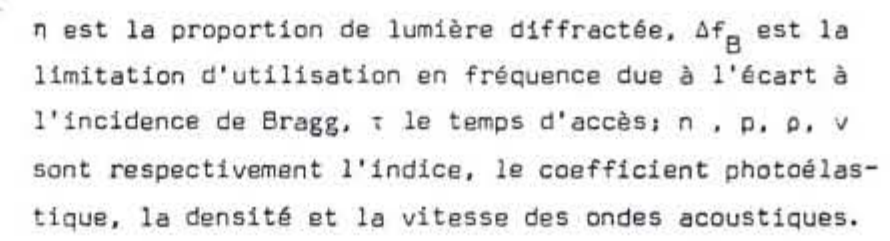

qui rend compte en plus de l'efficacité de diffraction de la bande passante de modulation. Un troisième facteur de mérite apparait aussi, le facteur $\mathrm{M}_{3}$

$M_{3}=\frac{n^{7} p^{2}}{\rho v_{a}^{2}}$

qui en outre rend compte du temps d'accès.

Les modulateurs de Bragg sont capables de fonctionner comme déflecteurs de faisceau ou comme commutateurs puisque le faisceau de sortie fait un angle différent du faisceau d'entrée.

Si la fréquence de l'onde acoustique est maintenue constante, le faisceau peut être commuté de $2 \theta$ en appliquant une puissance acoustique suffisante pour produire $100 \%$ de diffraction de l'ordre 0 dans l'ordre 1. 


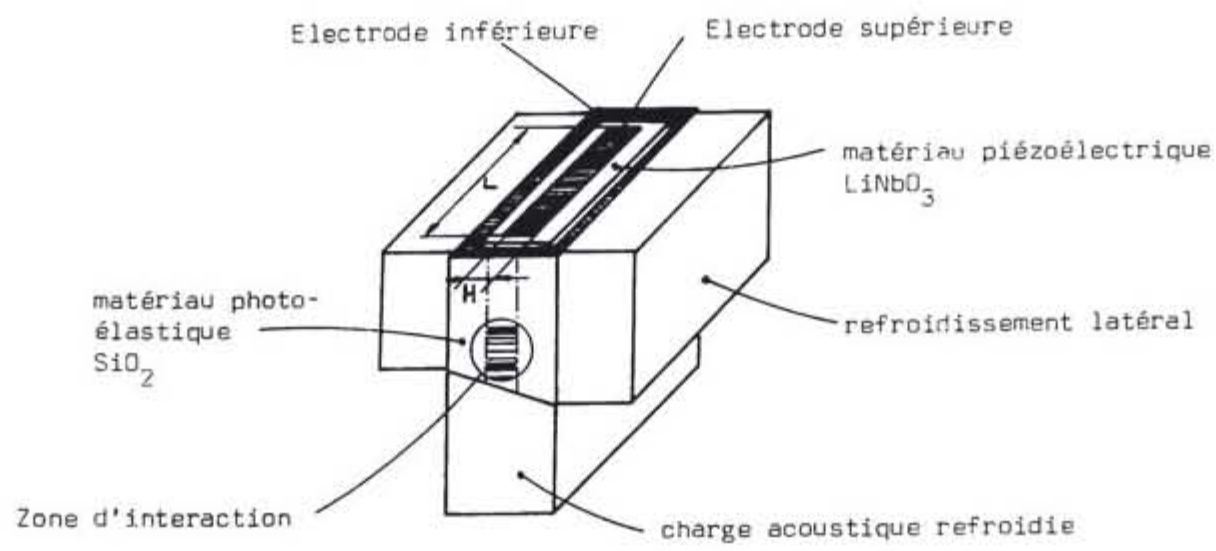

Les modulateurs de lumière réalisent le contrôle de l'intensité du faisceau diffracté; il suffit de moduler en HF le transducteur piézoélectrique et occulter la partie du faisceau non diffractée. Les critères sont ici la bande de fréquence de modulation et la cadence qui peut être limitée par le temps d'accès (temps de parcours de l'onde acoustique dans le faisceau lumineux: typiquement $1,68 \mu \mathrm{s} / \mathrm{cm}$ pour la silice). Pour améliorer cettte dernière caractéristique le faisceau lumineux est généralement focalisé dans le modulateur acousto-optique.

Par ailleurs on peut faire varier la fréquence de l'onde acoustique pour modifier l'angle de déflexion.

Dans les applications à la déflexion, un paramètre important est le nombre $\mathrm{N}$ de points résolus. On peut montrer que $\mathrm{N}$ est de l'ordre de :

$N=\frac{2 \Lambda b n}{\lambda L}$

où b est la largeur du faisceau incident.

Un autre paramètre important du déflecteur est la bande passante du signal acoustique qu'il peut accepter. On peut montrer qu'elle est égale à

$\Delta f=2 v_{a} \Lambda \lambda L$

Il en résulte que $N=\Delta f t$ où t désigne le temps de transit de l'onde acoustique à travers le faisceau de largeur $\mathrm{b}\left(t=b / v_{a}\right)$ En pratique très souvent la réponse est limitée par la réponse du transducteur piézoélectrique qui sert à générer l'onde acoustique. 
Il faut noter qu'il existe un compromis entre le nombre de poits résolus $\mathrm{N}$ et le temps d'accès, puique le temps de transit peut être diminué en réduisant $b$, mais du coup il y a moins de points résolus.

Ordre de grandeur:

$\Delta f=1 \mathrm{GHz}$; pour avoir $\mathrm{N}=1000$, il faut $\mathrm{t}=1 \mu \mathrm{s}$ et $\mathrm{b}=3,4 \mathrm{~mm}$ en supposant $\mathrm{v}_{\mathrm{a}}=3,4 \times 10^{3} \mathrm{~m} / \mathrm{s}$.

\section{3 - EFFETS MAGNETO-OPTIQUES}

\section{3-A - EFFET FARADAY}

L'effet magnéto-optique principal, l'effet Faraday, est très important dans les grenats ferrimagnétiques tels les grenats d'yttrium et de fer (Yttrium Iron Garnet ou YIG) et ses dérivés. En effet, cet effet consiste en une rotation indépendante du sens de propagation, de la polarisation d'un faisceau lumineux; il est couramment d'une centaine de degrés par $\mathrm{cm}$ et peut suivant les matériaux et la longueur d'onde atteindre plusieurs dizaines de milliers de degrés par $\mathrm{cm}$. Le YIG et ses substitués ont une fenêtre de transparence située entre 1,1 et 4,5 $\mu \mathrm{m}$.

$\mathrm{Si}$ on considère un milieu supposé infini d'aimantation $\mathrm{M}$, les deux principaux effets décrivant l'interaction entre une onde optique et $\mathrm{M}$ sont l'effet Faraday et l'effet Cotton Mouton.L'effet Faraday concerne l'interaction de la lumière et la composante de $\mathrm{M}$ suivant la direction de propagation; l'effet Cotton - Mouton correspond a l'interaction avec la composante normale à la propagation.

Pour une propagation perpendiculaire a l'aimantation seules interviennent les interactions du second ordre. Nous ne le traiterons pas ici.

Les interactions du premier ordre se traduisent en milieu uniformément aimanté par une birefringence circulaire. En effet les modes propres ont des polarisations circulaires contradirectives auxquelles correspondent les indices de réfraction: 
$n_{ \pm}=\sqrt{\varepsilon_{r}} \pm \frac{A M_{z}}{2 \sqrt{\varepsilon_{r}}}$

où $\mathrm{M}_{z}$ est la composante de l'aimantation dans la direction de propagation de la lumière. On suppose une onde incidente polarisée linéairement; après avoir parcouru une distance L, ses composantes propres contrarotatives qui se propagent avec des vitesses différentes sont déphasées et le champ résultant qui est aussi polarisé linéairement subit une rotation $\theta_{F}$ égale à:

$\theta_{F}=\frac{\pi A M_{z} L}{\lambda \sqrt{\varepsilon_{r}}}$

$\theta_{F}$ est la rotation de Faraday, $\lambda$ est la longueur d'onde.

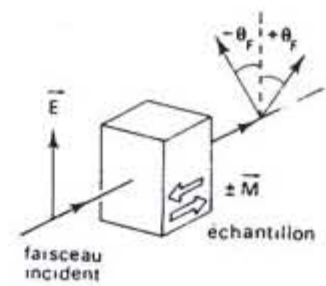

Une caractéristique importante de cette rotation est sa non réciprocité: l'inversion du sens de propagation ne change pas le sens de rotation. Pour le YIG à $1,15 \mu \mathrm{m}$ la rotation de Faraday est de $263 \%$; ceci correspond à un écart de l'ordre de $10^{-4}$ entre les indices des deux polarisations circulaires contrarotatives.

\section{3-B - APPLICATIONS : ISOLATEUR OPTIQUE}

Un isolateur permet de protéger la source de lumière des réflexions parasites; ces réflexions sont bien connues pour rendre instable l'émetteur laser. Avec le développement des communications par fibre optique, ces composants deviennent de plus en plus utiles pour protéger la diode laser à semiconducteur des réflexions provenant des divers composants optiques situés tout le long de la liaison.

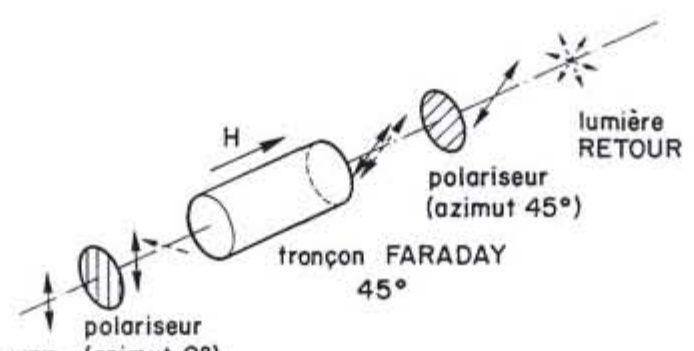

lumière ALLER (azimut $0^{\circ}$ ) 
Un isolateur est composé d' un tronçon non réciproque et de deux polariseurs correctement orientés comme l'indique la figure précédente.

Dans la section non réciproque constituée d'un tronçon de YIG le plan de rotation de la lumière tourne de $45^{\circ}$ par effet Faraday. Toute lumière réfléchie aura après un second passage dans ce tronçon une polarisation croisée avec le polariseur d'entrée. L'isolation est donc obtenue .

Un isolateur magnéto-optique fonctionnant à $1,3 \mu \mathrm{m}$ ou $1,5 \mu \mathrm{m}$ est constitué d'un cristal de YIG de 1 a $5 \mathrm{~mm}$ de long soumis à l'aide d'un aimant $\mathrm{Sm}$-Co à un champ magnétique supérieur à 2000 Oe qui aligne l'aimantation parallelement à la direction de propagation de la lumière. Les deux extrêmités du YIG sont traitées antireflet.

Typiquement les pertes totales sont de l'ordre de $1 \mathrm{~dB}$ et le taux d'isolation est $>$ à $25 \mathrm{~dB}$. Ces dispositifs utilisent du YIG pour l'IR et des verres paramagnétiques pour le domaine visible. Il existe même maintenant des isolateurs de structure optique plus complexe qui sont indépendants de la polarisation de la lumière.

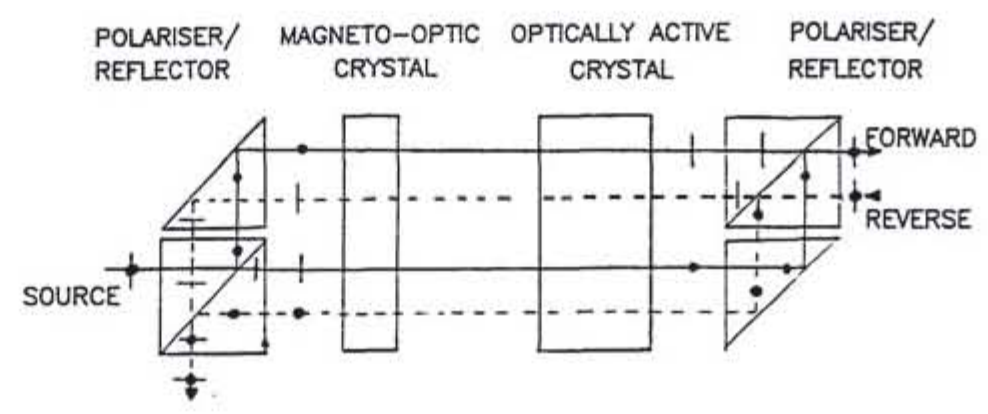

Le faisceau incident est décomposé en deux polarisations orthogonales par le polariseur d'entrée. Les deux polarisations sont ensuite traitées séparément suivant la méthode décrite précédemment avant d'être recombinées par le polariseur de sortie.

Ces dispositifs sont d'autant plus indispensables que les sources lasers semiconducteurs sont plus cohérentes (laser DFB monofréquence); deux isolateurs en série procurant des taux d'isolation voisins de $70 \mathrm{~dB}$ sont parfois nécessaires. Pour ces applications, les isolateurs sont 
directement munis de fibres amorces monomodes; ils emploient des microoptiques pour assurer un couplage optique efficace. Les pertes d'insertion restent $<1-2 \mathrm{~dB}$ et le taux d'isolation $>25$ dB pour un élement.

4 - ONDE OPTIQUE GUIDEE

\section{4-A-GENERALITES}

L'idée de base est de guider la lumière dans un film mince transparent déposé sur un substrat afin de s' affranchir de la diffraction. Il est ainsi possible de faire propager sur la longueur voulue un faisceau de taille très réduite sans qu'il ne diverge. Pour cela il suffit que la lumière se propage dans un matériau dont l'indice de réfraction est supérieur à celui des milieux environnants. Etant donné que les caractéristiques des composants sont d'autant meilleurs que les faisceaux guidés ont une section faible favorisant une interaction efficace avec la perturbation appliquée, il est préférable de ne considèrer que des guides monomodes, associables à des fibres monomodes qui elles aussi ont de meilleures performances que les fibres multimodes.

Les sections typiques de tels guides monomodes sont de quelques $\mu \mathrm{m}^{2}$ et les différences d'indice de réfraction vont de $10^{-3} \mathrm{a} 10^{-1}$. Nous allons voir qu'un guide monomode est conçu en jouant à la fois sur les épaisseurs des couches et leurs indices de réfraction respectifs.

\section{4-B - PROPAGATION GUIDEE MONOMODE ET MULTIMODE}

\section{4 - B - 1 - Modes TE et TM d'un guide plan monocouche}

Considérons la propagation d'une onde optique monochromatique de pulsation $\omega$ se propageant suivant l'axe $\mathrm{z}$ : 


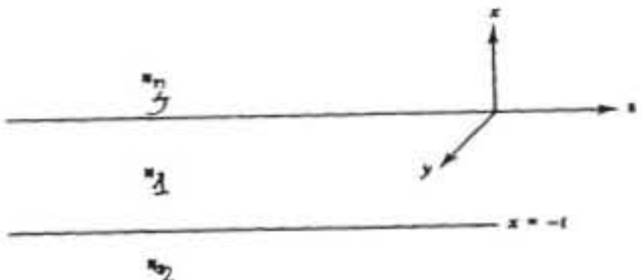

$\vec{E}=\vec{E}(x, y) \exp -i(\omega t-\beta z)$

$\vec{H}=\vec{H}(x, y) \exp -i(\omega t-\beta z)$

où $\beta=\frac{2 \pi}{2} n=k n$ est la constante de propagation.

La structure est constituee de couches minces planes isotropes, sans perte et sans charge de courant (permittivite $\mu_{0}$ )

Des équations de Maxwell, on peut déduire l'équation de Helmholtz valable dans chacun des milieux $\mathrm{j}(=1,2,3)$

$\nabla^{2} \vec{E}+k^{2} n_{j}^{2} \vec{E}=0$

Les milieux étant semi-infinis dans la direction $y\left(\frac{\partial}{\partial y}=0\right)$, on peut montrer qu'il existe deux types de solution indépendantes au problème:

* les modes TE dont les seules composantes non nulles sont $\mathrm{E}_{y}, \mathrm{H}_{x}$ et $\mathrm{H}_{x}$;

* et les modes TM dont les seules composantes non nulles sont $E_{x}, E_{x}$ et $H_{y}$.

La condition de guidage dans le milieu 1 s'écrit en imposant l'existence d'une solution sinusoïdale dans le milieu 1 avec évanescence des champs dans les milieux 2 et 3 . II en résulte que l'indice effectif des modes guidés possibles doit saticf:itz simuitanément:

$$
\begin{aligned}
n & \geq n_{3} \\
\{n & \leq n_{1} \\
n & \geq n_{2}
\end{aligned}
$$

En supposant que $n_{1}>n_{2}>n_{3}$, les solutions mathématiques sont illustrées sur le diagramme de la figure suivante.

où 
(a) n'est pas une solution physique posssible

(b) et (c) sont des modes guidés bien confinés $\left(\mathrm{TE}_{0}, \mathrm{TE}_{1}, ..\right)$

(d) est un mode de substrat

(e) est un mode d'air

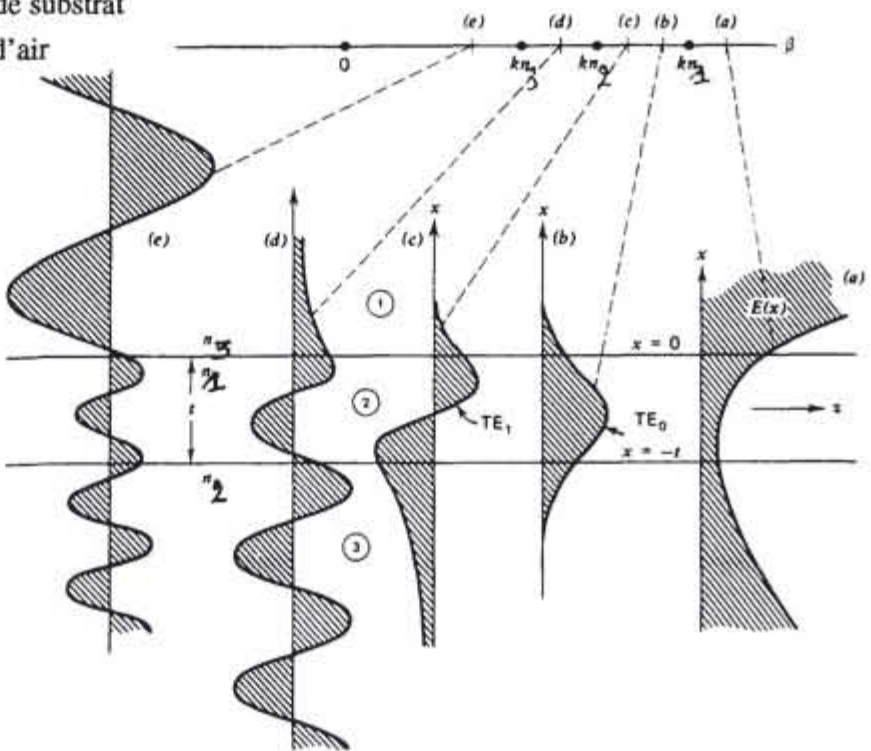

La condition de guidage s'exprime donc par:

$n_{1} \geq n \geq n_{2} \geq n_{3}$

L'équation modale des modes TE (resp. TM) résulte de l'écriture de la continuité des composantes tangentielles des champs $\mathrm{E}$ et $\mathrm{H}$ aux interfaces 3-1 et 1-2.

$t=\frac{1}{\sqrt{n_{1}^{2}-n^{2}}}\left\{m \pi+\tan ^{-1}\left[\eta_{1} \sqrt{\frac{n^{2}-n_{2}^{2}}{n_{1}^{2}-n^{2}}}+\tan ^{-1}\left[\eta_{2} \sqrt{\frac{n^{2}-n_{3}^{2}}{n_{1}^{2}-n^{2}}}\right\}\right.\right.$

avec: $\quad * \eta_{1}=\eta_{2}=1$ pour TE

$$
* \eta_{1}=\left(\frac{n_{1}}{n_{2}}\right)^{2} \quad \eta_{2}=\left(\frac{n_{1}}{n_{3}}\right)^{2} \text { pour TM }
$$

Il apparaît donc une relation transcendante entre l'indice effectif du mode propagé et les paramètres du guide $\left(t, n_{1}, n_{2}, n_{3}\right)$. Elle montre que le guidage dans le milieu 1 ne peut se faire que pour certaines valeurs discrètes de $\mathrm{n}$; $\mathrm{m}$ désigne le numéro du mode correspondant (il indique également le nombre de zéros de la fonction champ électrique). 
Le schéma illustrant le comportement général des solutions de l'équation modale fait apparaître des épaisseurs de coupure pour les différents modes $\mathrm{TE}_{\mathrm{m}}$ et $\mathrm{TM}_{\mathrm{m}}$. Cette épaisseur correspond à la limite du confinement dans le milieu 1, c'est à dire quand $\mathrm{n}=\mathrm{n}_{2}$.

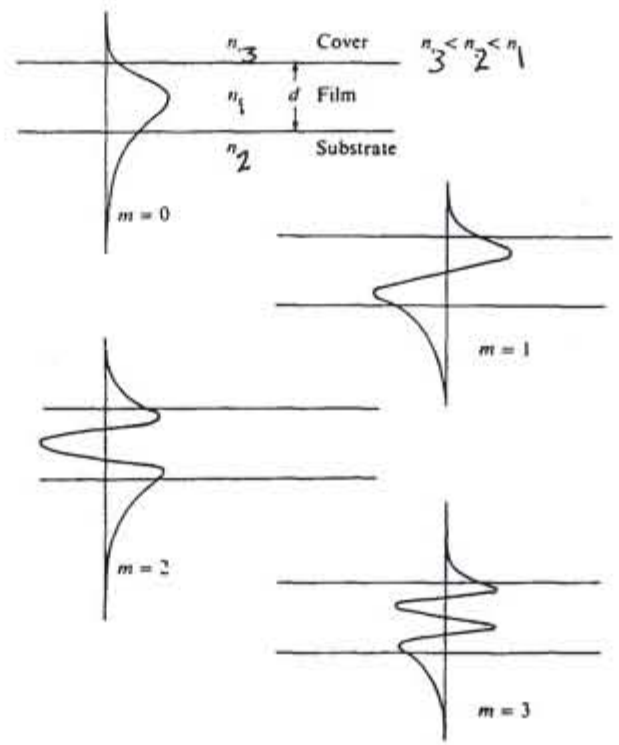

La figure illustre le profil de champ des modes guides d'ordre différent. On peut noter qu'une partie notable de la lumière se propage à l'extérieur de la couche 1 . On définit pour quantifier ce phénomène le facteur de confinement du mode comme étant le rapport entre la puissance guidée dans la couche 1 et la puissance totale du mode. Il est donc compris entre 0 et 1 .

Il est pratique de normaliser les équations de dispersion en définissant:

*la frequence normalisée: $v=\frac{2 \pi}{\lambda} t \sqrt{n_{1}^{2}-n_{2}^{2}}$

* la constante de propagation normalisée: $b=\frac{n^{2}-n_{2}^{2}}{n_{1}^{2}-n_{2}^{2}}$

* le paramètre d'asymétrie: $a=\frac{n_{2}^{2}-n_{3}^{2}}{n_{1}^{2}-n_{2}^{2}}$ a= 0 pour un guide symétrique et $+\infty$ pour un guide où $n_{1} \approx n_{2}>n_{3}$. 
L'équation modale peut ainsi s'écrire dans le cas TE:

$v \sqrt{1-b}=m \pi+\tan ^{-1} \sqrt{\frac{b}{1-b}}+\tan ^{-1} \sqrt{\frac{a+b}{1-b}}$

Le cas TM peut se traiter de manière semblable en posant: $a_{T M}=\left(\frac{n_{1}}{n_{3}}\right)^{4} a$. Il se ramène à la même equation que TE quand $n_{1}-n_{2} \ll$.

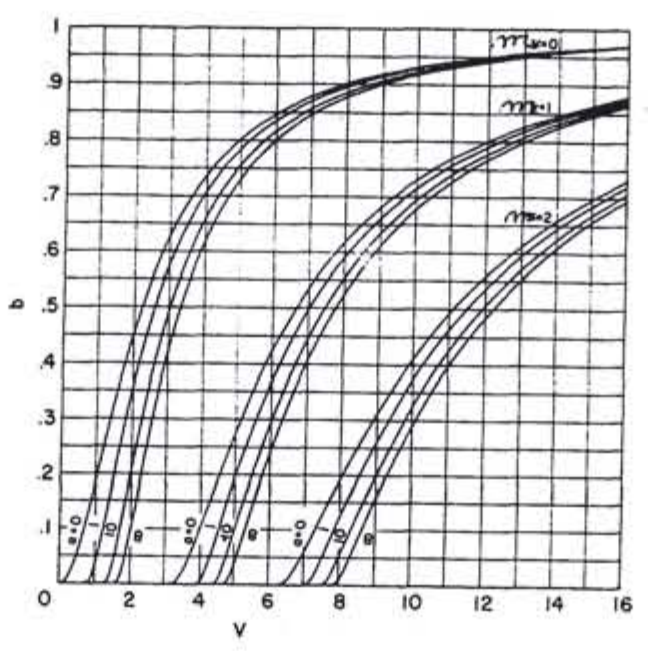

\section{Cas d'un profil d'indice quelconque:}

La résolution du problème est alors plus complexe et ne peut se faire analytiquement que pour quelques profils simples (linéaire, exponentiel,...).

Une méthode approximative très souvent employée est celle des multicouches qui consiste à remplacer le profil d'indice par une série de marches d'indice constant et d'utiliser le formalisme matriciel bien connu pour le calcul des systèmes optiques multicouches, rendant systématique la répétition des conditions aux limites aux interfaces. La précision dépend évidemment du nombre de marches utilisés. L'équation des modes cherchés est obtenue en annulant le déterminant du produit des matrices traduisant le passage d'une onde guidé à travers les multicouches. 
Une autre méthode approximative est également employée pour résoudre le cas de la propagation dans des milieux d'indice variable; c'est la méthode WKB largement discutée en mécanique quantique.

La condition qui va permettre de calculer les valeurs des indices n des modes pouvant se propager est alors:

$k \int_{0}^{x_{m}}\left\{n(x)^{2}-n\left(x_{m}\right)^{2}\right\} d x-2 \Phi_{1}-2 \Phi_{2}=2 m \pi$

où $x_{m}$ est le "point tournant" du mode d'ordre m pour lequel $n\left(x_{m}\right)=n_{m}$.
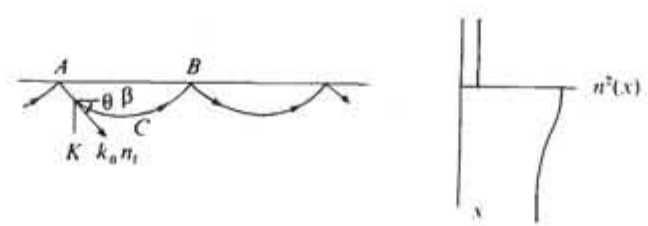

C'est en ce point que la solution passe d'une allure oscillatoire à une allure évanescente. et où $-2 \phi_{1}$ et $-2 \phi_{2}$ représentent les déphasages au point tournant et à la surface du guide.

En considérant le problème WKB inverse, cette équation permet de reconstruire un profil d'indice inconnu à partir de la mesure des indices des modes propagés par une structure. Quand les milieux ont des profils simples, la méthode conduit à une résolution exacte; quand ils sont inhomogènes, la résolution ne peut être que numérique.

\section{4 - B - 2 - Guide à 2 Dimensions. Méthode des indices effectifs}

Il n'y a pas de solution analytique exacte au probleme de propagation dans une structure à 2 dimensions comme celle illustrée sur la figure .

Comme en général les différences d'indice relatives sont faibles ( $<5 \%$, on peut simplifier le traitement en supposant que la polarisation de la lumière est identique à celle d'un guide plan. Cette approximation permet de considérer le probleme vectoriel a l' aide d'une équation scalaire.

Une des méthodes les plus utilisées pour analyser les guides à deux dimensions est la méthode dite des indices effectifs. Le principe illustré sur la figure suivante, consiste à ramener le problème à deux dimensions à la résolution de deux problèmes à une dimension. 

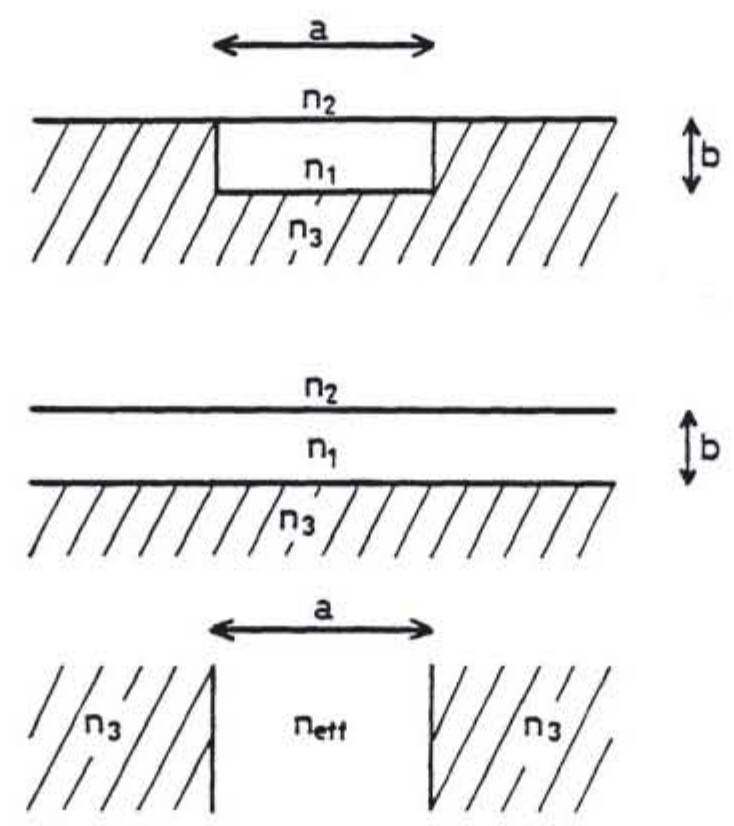

Principe de la méthode des indices effectifs

Supposons que $n_{1}>\sim n_{2} \gg n_{3}$. On commence par calculer l'indice effectif du guide plan d'épaisseur égale à celle du guide ruban; on peut connaitre exactement la valeur de l'indice effectif $n_{\text {eff }}$ dans le cas des deux polarisations qui sont alors purement TE ou TM. On calcule ensuite les constantes de propagation du guide plan d'épaisseur égale à celle du guide ruban et

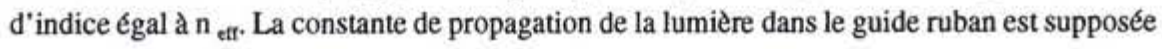
égale à celle du guide plan équivalent ainsi obtenu.

\section{4- B - 3 - Modes couplés}

Un grand nombre de composants (coupleur directif, convertisseur de modes TE $\Leftrightarrow=T M$, modes diffractés par un réseau,..) implique le couplage entre modes.

Les modes étant supposés sans perte et non perturbés par le couplage (faible couplage). le formalisme des modes couplés conduit au système d'équations suivantes:

$d A / d z=K B \exp (j \Delta \beta z)$

$d B / d z=K A \exp (-j \Delta \beta z)$ 
où $\mathrm{A}$ et $\mathrm{B}$ sont les amplitudes des deux modes couplés codirectifs, $\Delta \beta$ représente la différence entre leur constantes de propagation et $\mathrm{K}$ est le coefficient de couplage.

La solution de ce système différentiel conduit pour le taux de conversion défini par $R(z)=I_{A}(z) / I_{A}(0)$ a l'expression:

$R(z)=\frac{4 K^{2}}{4 K^{2}+\Delta \beta^{2}} \sin ^{2}\left\{\left(4 K^{2}+\Delta \beta^{2}\right)^{1 / 2} \frac{z}{2}\right\}$

où $\mathrm{A}(\mathrm{z})$ est l'intensité de l'onde $\mathrm{A}$ en $\mathrm{z}$.

Le taux de conversion maximal $\mathrm{R}_{\mathrm{M}}$ vaut:

$R_{M}=\frac{4 K^{2}}{4 K^{2}+\Delta \beta^{2}}$

Cette relation montre que pour $K$ constant la conversion n'est complete que si $\Delta \beta=0$. Dans ce cas on obtient une conversion complète pour une distance $\mathrm{z}_{0}$ telle que $z_{0}=\pi /(2 K)$.

Alors,

$$
\begin{aligned}
& I_{A}(z)=\sin ^{2} K z \\
& I_{B}(z)=\cos ^{2} K z
\end{aligned}
$$

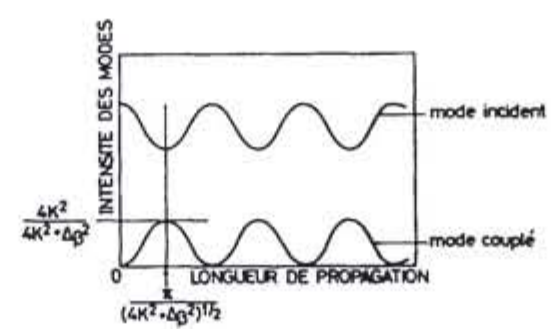

\section{4- B - 4 - Propagation dans une fibre}

a) Definition .

Une fibre optique est un guide diélectrique cylindrique, qui possede (sauf cas exceptionnels comme celui des fibres à maintien de polarisation) une symétrie cylindrique autour de son axe de révolution : en particulier, l'indice de réfraction ne dépend que de la distance à l'axe $r$.

De manière générale,une fibre est constituée par :

- une zone guidante centrale, où sera confinée la plus grande partie de l'énergie lumineuse, appelée coeur de la fibre.

- une gaine optique d'indice de refraction inférieur à celui du coeur, et ou ne se propagera 
qu'une onde évanescente.

- une ou plusieurs gaines de protection destinées à protéger le guide optique et à lui conférer de meilleures propriétés mécaniques.

Pour les fibres en silice, le diametre extérieur $\phi_{e x t}$ est souvent de l'ordre de $250 \mu \mathrm{m}$ tandis que le diamètre de la gaine optique $\phi_{g}$ est standardisé a $125 \mu \mathrm{m}$ dans la majorité des cas. Le diamètre de coeur dépend, quant à lui, du type de fibre considéré (multimode ou monomode).

b) Profils d'indice de réfraction .

On distingue d'après le type de profil d'indice $n=f(r)$ deux familles de fibres optiques: les fibres à saut d'indice ou à gradient d'indice.

En pratique, pour les fibres optiques en silice, l'écart relatif d'indice de réfraction entre le coeur et la gaine optique est faible $\left(10^{-2}\right.$ et $\left.10^{-3}\right)$.

Il existe des profils d'indice plus complexes. Dans le cas des fibres à gradient d'indice, on rencontre très souvent des profils d'indice modélisables par la relation :

$$
n^{2}(r)=n_{1}^{2}\left(1-2 \Delta\left(\frac{r}{a}\right)^{\alpha}\right)
$$

avec : $a=\phi_{c} / 2$ rayon de coeur, et :

$$
\Delta=\frac{1}{2}\left(\frac{n_{1}^{2}-n_{2}^{2}}{n_{1}^{2}}\right)
$$

soit encore :

$$
n_{2}^{2}=n_{1}^{2}(1-2 \Delta)
$$

Dans la plupart des cas, $n_{1}$ et $n_{2}$ sont très voisins, et $\Delta$ est petit devant l'unité . On a donc, en développant au premier ordre l'expression de $n(r)$ :

$$
n(r)=n_{1}\left(1-\Delta\left(\frac{r}{a}\right)^{\alpha}\right)
$$

et le paramètre $\Delta$ se confond avec la variation relative d'indice :

$$
\Delta=\frac{n_{1}-n_{2}}{n_{1}}
$$

c) Fibres monomodes, fibres multimodes. 
Comme les guides d'ondes planaires, les fibres optiques admettent, dans le cas général, un certain nombre de modes de propagation. Ces modes sont des configurations particulières du champ électromagnétique susceptibles de se propager dans la fibre avec une constante de propagation $\beta$ caractéristique du mode considéré :

$$
\begin{aligned}
\vec{E}(r, \theta, z, t) & =\vec{E}_{l}(r, \theta) e^{j(\theta, r-\infty)} \\
\vec{H}(r, \theta, z, t) & =\vec{H}_{l}(r, \theta) e^{j(\theta, t-\infty)}
\end{aligned}
$$

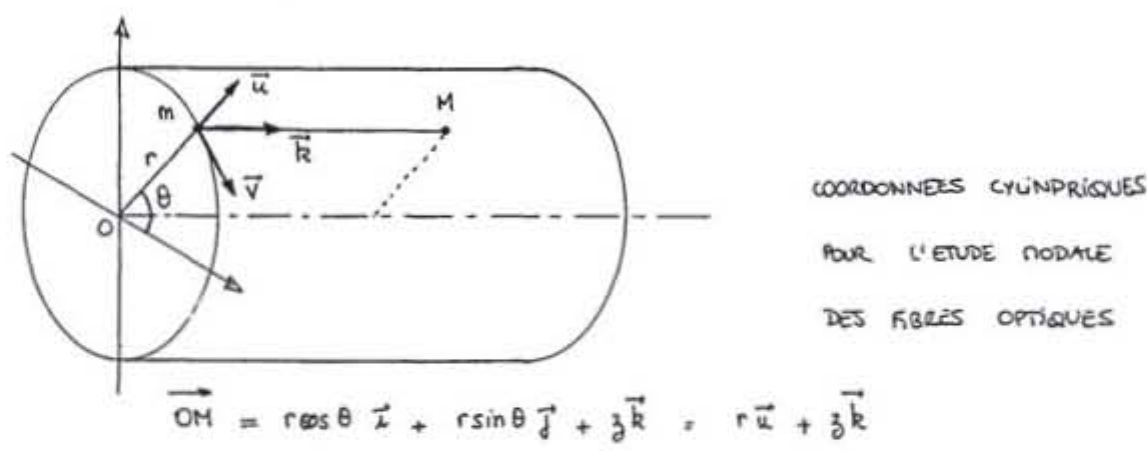

La distribution d'amplitude du champ caractéristique du mode $\mathrm{n}$ " 1 est déterminée par les deux fonctions : $\vec{E}_{B}(r, \theta)$ et $\vec{H}_{B}(r, \theta)$ dont la forme dépend du mode considéré

Lorsque l'on diminue le diamètre de coeur d'une fibre, ou son écart relatif d' indice $\Delta$, le nombre de modes permis diminue. En fait, comme on l'a vu à propos du guide plan, le paramètre qui détermine le nombre de modes d'une fibre est le paramètre normalisé $\mathbf{V}$ :

$$
V=\frac{2 \pi}{\lambda} a \sqrt{n_{1}^{2}-n_{2}^{2}}
$$

avec : a : rayon de coeur de la fibre

$\mathrm{n}_{1}$ : indice maximal du coeur (obtenu sur l'axe de la fibre)

$\mathrm{n}_{2}$ : indice de la gaine optique 
$\mathrm{V}$ qui est une grandeur sans dimension est appelée fréquence normalisée, ou rayon de coeur normalise. En dessous d'une certaine valeur de V, la fibre n'admet plus qu'un seul mode de propagation : on parle alors de fibre monomode.

- la condition pour qu' une fibre à saut d'indice soit monomode s'écrit :

$$
0<V \leq 2,405
$$

- lorsqu'une fibre à gradient d'indice admet un grand nombre de modes, celui-ci peut être estimé par la relation approchee :

$$
N_{G I}=\frac{\alpha}{\alpha+2} \frac{V^{2}}{2}
$$

cette relation peut être généralisée au cas d'une fibre à saut d'indice en faisant tendre le paramètre $\alpha$ du profil vers l'infini:

$$
N_{S I}=\frac{V^{2}}{2}
$$

Dans le cas de fibres à saut d'indice fortement multimodes ( $V$ et $N$ grands ), l'approximation de l'optique géométrique permet d'associer à chaque mode une valeur de l'angle d'incidence i d'un rayon méridien (situé dans un plan contenant l'axe de la fibre) sur le dioptre coeur-gaine. D'après la théorie électromagnétique, les modes guidés - pour lesquels l'énergie reste confinée au voisinage du coeur - sont en nombre fini .

Les modes d'ordre supérieur sont caractérisés par une certaine valeur de l'angle d'incidence i - comprise entre $\pi / 2$ et l'angle limite de réflexion totale $i_{1}$ - d'autant plus grande que l'ordre du mode est plus élevé.

En pratique, pour des guides de "grand" rayon de coeur ( quelques dizaines de microns pour les fibres courantes ), les modes permis sont très nombreux et les valeurs permises de l'angle i forment pratiquement un continuum, ce qui permet de faire le lien avec l'optique géométrique où aucune "règle de sélection" n'existe pour l'angle d'incidence $i$.

Il est à noter que le trajet parcouru à l'intérieur de la fibre par un rayon dépend de l'angle i, donc du mode considéré : ce résultat est d' ailleurs valable même en dehors de l'approximation géométrique de la propagation par rayons. 
Cette différence entre les trajets relatifs aux divers modes de propagation de la structure guidante est à l'origine de la dispersion modale, qui est une des causes de distorsion du signal dans les transmissions par fibre.

Ce phénomène se traduit par un élargissement temporel de toute impulsion lumineuse se propageant dans la fibre. Il est d'autant plus important que le nombre de modes guidés est plus grand, ce qui justifie l'interêt portê aux fibres optiques monomodes pour les systèmes de transmission optique.

d) Angle d'acceptance. Ouverture numérique .

Considérons une fibre multimode à saut d'indice, dans l'approximation de l'optique géométrique (propagation par rayons):
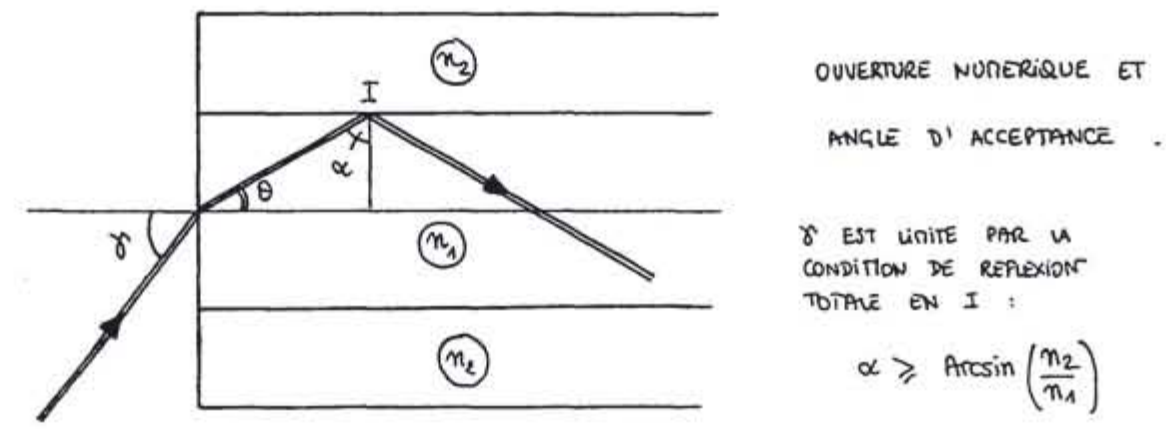

L'ouverture numérique de la fibre est définie par :

$$
\text { O.N. }=\sin \gamma_{\max }=\sqrt{n_{1}^{2}-n_{2}^{2}}
$$

et son angle d'acceptance est défini par :

$$
\gamma_{\max }=\arcsin \sqrt{n_{1}^{2}-n_{2}^{2}}
$$

En pratique, les indices de coeur et de gaine sont très voisins et :

$$
O . N . \approx n_{1} \sqrt{2 \Delta}
$$


Ilest à noter que ces expressions basées sur les lois de l'optique géométrique ne sont plus valables lorsque le rayon de coeur devient trop faible (typiquement inférieur à $5 \mu \mathrm{m}$ ). D'autre part, les rayons considérés pour le calcul de l'ouverture numérique étaient des rayons méridiens. On peut montrer que ceci conduit à sous-estimer légèrement l'ouverture numérique de la fibre

En ce qui concerne les fibres à gradient d'indice, le problème du calcul de l'ouverture numérique est plus complexe : puisque l'indice de coeur n'est pas constant, la valeur de l'angle d'acceptance dépendra du point d'incidence du rayon sur la face d'entrée de la fibre. Cependant, dans la plupart des cas, on obtiendra une estimation approximative de l'ouverture numérique par la relation précédente.

L'ouverture numérique permet de chiffrer l'aptitude d'une fibre optique à collecter la lumière. Supposons qu'on focalise sur la face d'entrée d'une fibre un faisceau conique de demi-angle au sommet $2 \phi$ :

${ }^{*} \mathrm{Si} \phi<\gamma_{\max }$, toute la lumière incidente sera couplée dans la fibre, aux pertes par réflexion près.

* si $\phi>\gamma_{\max }$, seule la fraction de l'énergie incidente contenue dans le cône de demi-angle au sommet $\gamma_{\max }$ pourra être couplée.

\section{e) PROPAGATION DANS LES FIBRES : ETUDE MODALE}

L'approximation de l'optique géométrique, qui considère des rayons lumineux se propageant dans la fibre, ne peut plus être utilisée lorsque le rayon de coeur n'est plus très grand devant la longueur d'onde. La propagation doitêtre étudiée à partir des équations de MAXWELL. Elle ne conduit pas - en général - à des solutions analytiques. Dans le cas de la structure de fibre la plus simple et moyennant plusieurs hypothèses simplificatrices, on arrive à exprimer sans trop de difficultés les différentes composantes des champs à partir de fonctions de BESSEL d'ordre entier.

\section{* Fibre à saut d'indice.}

Pour résoudre les équations de MAXWELL dans une fibre, il est intéressant d'utiliser les coordonnées cylindriques $(r, \theta, z)$ définies par la figure précédente.

On peut montrer que les équations de MAXWELL écrites dans le système de coordonnées cylindriques définies ci-dessus peuvent être combinées pour obtenir deux équations différentielles relatives à $\mathrm{Hz}$ et $\mathrm{Ez}$ : 


$$
\begin{aligned}
& \frac{\partial^{2} E_{z}}{\partial r^{2}}+\frac{1}{r} \frac{\partial E_{z}}{\partial r}+\frac{1}{r^{2}} \frac{\partial^{2} E_{z}}{\partial \theta^{2}}+\kappa^{2} E_{z}=0 \\
& \frac{\partial^{2} H_{z}}{\partial r^{2}}+\frac{1}{r} \frac{\partial H_{z}}{\partial r}+\frac{1}{r^{2}} \frac{\partial^{2} H_{z}}{\partial \theta^{2}}+\kappa^{2} H_{z}=0
\end{aligned}
$$

En général, $\mathrm{E}_{2}$ et $\mathrm{H}_{2}$ sont simultanément non nuls : il n'existe pas de modes TE et TM comme dans le cas du guide plan. La solution des équations différentielles relatives a $\mathrm{E}_{2}$ et $\mathrm{H}_{2}$ est recherchée sous la forme:

$$
\Psi(r, \theta, z, t)=f(r) \cdot g(\theta) \cdot e^{j(\omega x-\beta z}
$$

et on montre alors que

$$
g(\theta)=e^{ \pm j l \theta}
$$

où $1=0,1,2, \ldots$ etc

Il est nécessaire que la fonction g soit périodique de période $2 \pi$ donc que 1 soit un nombre entier.

D'autre part, la partie radiale $f(r)$ doit satisfaire à l'équation différentielle :

$$
\frac{d^{2} f}{d r^{2}}+\frac{1}{r} \frac{d f}{d r}+\left(\frac{\omega^{2} n^{2}}{c^{2}}-\beta^{2}-\frac{l^{2}}{r^{2}}\right) f=0
$$

avec : $\mathrm{n}=\mathrm{n}_{1}$ dans le coeur $\left(\mathrm{r}<\frac{\phi_{\mathrm{c}}}{2}\right)$

$$
\mathrm{n}=\mathrm{n}_{2} \text { dans la gaine }\left(\mathrm{r}>\frac{\boldsymbol{t}}{2}\right)
$$

On peut montrer que les modes guidés de la fibre ont des constantes de propagation $\beta$ vérifiant, comme dans le cas du guide plan :

$$
\beta_{1}=\frac{n_{1} \omega}{c}>\beta>\frac{n_{2} \omega}{c}=\beta_{2}
$$

et que les champs prennent une forme "oscillatoire" dans le coeur, et "amortie" dans la gaine, qui ne sont toutefois pas sinusoïdales ni exponentielles comme dans le cas du guide plan, comme nous allons le voir.

$$
\begin{aligned}
u^{2} & =4 \beta_{1}^{2}-\beta^{2} \\
w^{2} & =\beta^{2}-\beta_{2}^{2}
\end{aligned}
$$

Afin de travailler avec des quantités sans dimension, on introduit souvent:

$$
\begin{aligned}
U & =\frac{d}{2} u \\
W & =\frac{d}{2} w
\end{aligned}
$$

où $\mathrm{d} / 2$ est le rayon de coeur de la fibre. 
U et W sont reliés au paramètre normalise :

$$
V=\frac{\omega}{c} d \sqrt{n_{1}^{2}}-n_{2}^{2}
$$

par la relation :

$$
V^{2}=U^{2}+W^{2}
$$

On peut montrer que les solutions du problème sont:

$$
f(r)=A \frac{J_{l}(2 U r / d)}{J_{l}(U)}
$$

si $r<d / 2$

$$
f(r)=A \frac{K_{l}(2 W r / d)}{K_{l}(W)}
$$

si $r>d / 2$

$\mathrm{J}_{1}(\mathrm{x})$ est la fonction de Bessel d'ordre entier 1

$K_{1}(x)$ est la fonction de Bessel modifié d'ordre entier 1 Ces fonctions sont tracées pour quelques valeurs de 1 .

On montre que $\mathrm{U}$ et $\mathrm{W}$ sont reliés par la relation :
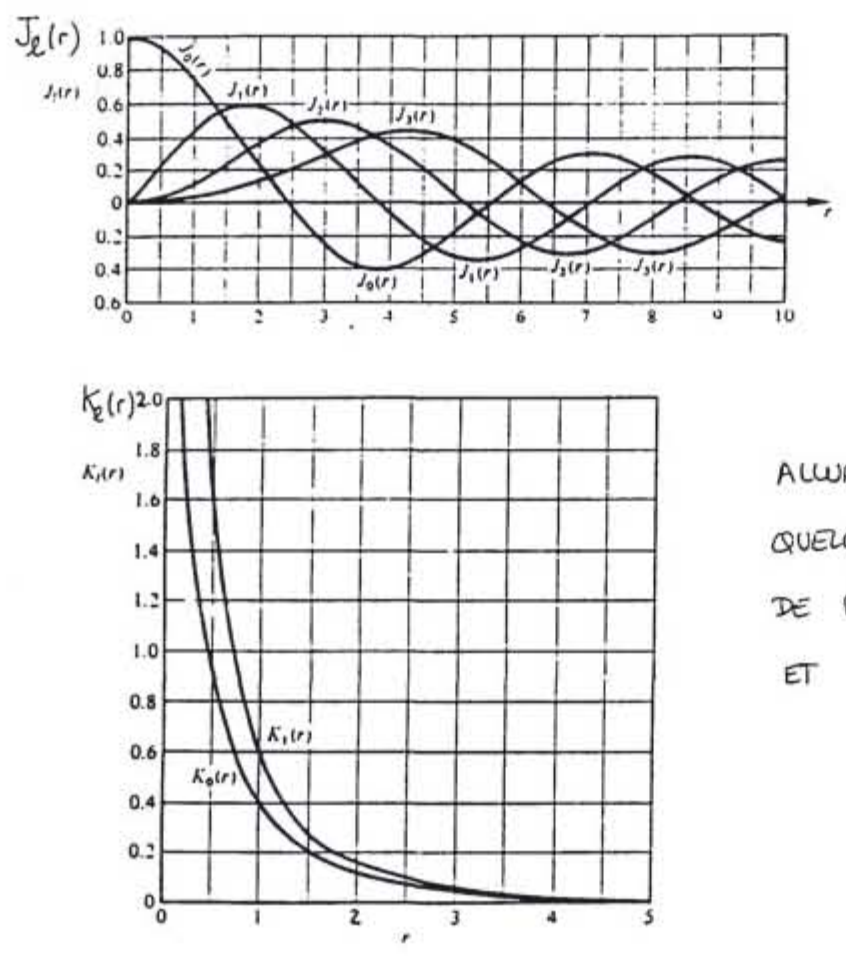

ALWRE DE

QUERQUES FONGTONS

DE BESSEL Je

ET Ke 


$$
U \cdot \frac{J_{l-1}(U)}{J_{l}(U)}=-W \frac{K_{l-1}(W)}{K_{l}(W)}
$$

Cette relation permet de déterminer $\mathrm{U}$ et $\mathrm{W}$, puisque ces deux paramètres sont en outre reliés par:

$$
U^{2}+W^{2}=V^{2}
$$

On a tenu compte pour établir ces relations, de la continuité de $\mathrm{E}_{\mathrm{z}}$ ou $\mathrm{H}_{\mathbf{z}}$ lorsque $\mathrm{r}=\mathrm{d} / 2$, à l'interface coeur gaine. On a en outre imposé que les amplitudes des champs tendent vers zéro pour $r$ grand ( guidage )

Comme dans le cas du guide plan, il est possible de faire une discussion graphique de la relation de dispersion. Pour une valeur donnée du nombre entier 1 , il y aura un nombre fini de solutions. La $\mathrm{n}^{\text {ieme }}$ solution correspond à ce qu'on appelle le mode $\mathrm{LP}_{\mathrm{a}}$ ( LP pour Linéairement Polarisé).

Si l'on définit la constante de propagation normalisée $b$ :

$$
b=\frac{\beta^{2} / \beta_{0}^{2}-n_{2}^{2}}{n_{1}^{2}-n_{2}^{2}}=\frac{W^{2}}{V^{2}}
$$

où $\mathrm{U}$ et $\mathrm{V}$ sont donnés par

$$
\begin{gathered}
W=V \sqrt{b} \text { et } \\
U=V \sqrt{1-b}
\end{gathered}
$$

il est possible de tracer en fonction de $\mathrm{V}$ les valeurs de la constante de propagation normalisée b pour les différents modes, comme le montre la figure. Le mode $\mathrm{LP}_{01}$ est le seul mode excité si $\mathrm{V}<2,405$ : il s'agit alors d'une fibre monomode.

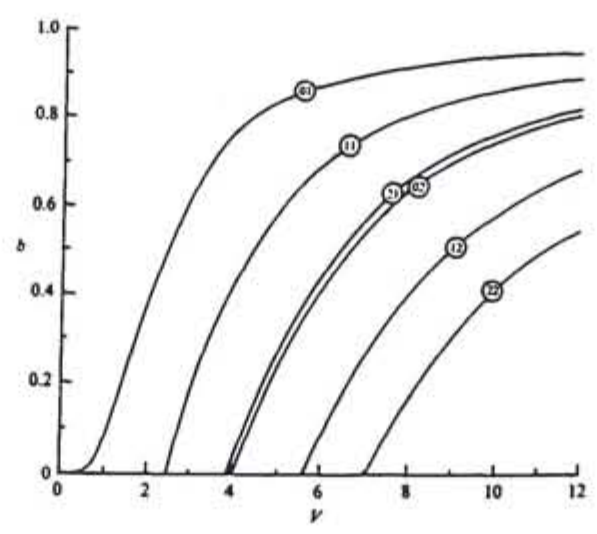


On peut considérer avec une excellente approximation que le mode propagé a une allure gaussienne:

$\psi(r)=A \exp \left(-\frac{r^{2}}{w^{2}}\right)$

quand $0,8<V<2,5$; dans ce cas,

$w / a=0,65+1,619 / V^{3 / 2}+2,879 / V^{6}$

\section{4 - C - VERS DE NOUVEAUX COMPOSANTS ACTIES: LES "OEIC"EN OPTIOUE INTEGREE}

C'est dans les années 70 qu'ont commencé les recherches dans une branche de l'optique qui allait prendre une grande expansion avec l'apparition de sources laser compactes et efficaces à base de matériaux semiconducteurs et avec les besoins crés par le développement de fibres optiques ayant une qualité de transmission exceptionnelle. L'expression Optique Intégrée a été forgée alors par analogie avec la technologie de l'Electronique Intégrée.

L'ambition est en effet de concevoir et de mettre en oeuvre des circuits optiques dont les composants actifs ou passifs sont fabriqués avec des processus planaires. Les éléments actifs peuvent être des lasers, des amplificateurs, des modulateurs, des matrices de commutation ou des détecteurs; les éléments passifs sont des filtres, des polariseurs, des diviseurs de puissance ou encore des miroirs. Les différents éléments du circuit sont ensuite interconnectés par des fibres optiques (intégration hybride), soit interconnectés par des guides de lumière fabriqués sur la même "puce" (intégration monolithique). De tels circuits offrent un gain très net en efficacité, en rapidité, en encombrement et en poids.

L'optique intégrée a un domaine privilégié d'application qui sont les liaisons par fibre optique monomode. Ce support permet en soi la transmission et le traitement de signaux très large bande sous forme multiplexée à la fois dans le temps et en longueur d'onde. On a donc besoin de composants pour traiter l'information sous sa forme optique, c'est à dire sans conversion en signaux électriques. Il existe d'autres domaines n'utilisant pas forcément l'optique, où l'optique intégrée peut néanmoins jouer un rôle important: il s'agit de l'instrumention où elle commence à remplacer certains capteurs moins performants, plus encombrants et plus sensibles aux perturbations électromagnétiques (capteurs de déplacement, capteurs de courant, gyroscope,...). L'optique intégrée étant étroitement liée aux fibres monomodes, les dispositifs doivent donc fonctionner dans les fenêtres de transmission des fibres en verre de silice $(0,8$ et surtout 1,3 et $1,5 \mu \mathrm{m}$ où les attenuations sont réduites à quelques dixièmes de $\mathrm{dB} / \mathrm{km}$ c'est à dire quasiment les valeurs theoriques).

Les technologies employées pour réaliser de tels dispositifs sont assez analogues à celles de la microélectronique. Les plus utilisées sont:

* l'épitaxie pour la croissance de couches minces de matériaux semiconducteurs monocristallins du type III-V (GaAs, InP et les alliages accordés sur ces matériaux)

* la diffusion ou l'implantation (diffusion localisée de $\mathrm{Ti}$ dans $\mathrm{LiNbO}_{3}$ pour faire les 
guides)

* l'échange d'ions $(\mathrm{Tl}, \mathrm{Ag}, \mathrm{K}, . .$.$) avec les ions \mathrm{Na}$ dans les verres, protons dans $\mathrm{LiNbO}_{3}$ pour fabriquer les guides

* l'evaporation ou la pulvérisation cathodique pour déposer des couches minces diélectriques (silice ou nitrure de silicium sur $\mathrm{Si}, .$. )

Pour obtenir les microstructures guidantes dans les couches minces, les techniques précédentes se combinent avec les opérations de photolithographie et de gravure.

Les critères de choix des matériaux sont les suivants:

* possibilité de fabriquer des guides monomodes à la longueur d'onde de propagation

* facteur de mérite élevé pour l'effet optoélectronique visé, par exemple recombinaison radiative maximum pour un émetteur, effet Pockels pour un modulateur, grande transparence pour un guide, absorption élevée pour un photodétecteur,..

* possibilité d'appliquer efficacement la perturbation pour un composant actif (jonction pn polarisée en inverse,contacts ohmiques ou redresseurs...).

Les matériaux les plus employés sont:

* les verres

* le silicium

* le niobate de lithium

* GaAs et les composés GaAlAs; InP et les alliages en GaInAsP.

Les verres ou le $\mathrm{Si}$ permettent de faire des guides optiques monomodes bon marché et à faible perte par échange ionique pour les premiers, par dépôt de silice, silice dopée ou nitrure de Si pour le second; ils ont le défaut majeur de ne conduire généralement qu'à des des composants passifs. Seule une perturbation thermique localisée modifiant l'indice de reffraction peut les rendre actifs, mais la réponse reste lente (>ms). Ils sont principalement utilisés soit comme diviseur de puissance de 1 vers $\mathrm{N}(\leq 16)$, soit comme interféromètre pour le filtrage en longueurd'onde, soit comme capteur

Le niobate de lithium dans lequel des guides à très faible perte $(<0,2 \mathrm{~dB} / \mathrm{cm})$ sont obtenus. par diffusion localisée de Ti a atteint un niveau de développement important du fait de l'effet Pockels. Le degré de confinement important de la lumière dô au guidage monomode permet d'obtenir sur une grande longueur des interactions transverses fortes avec des signaux de commande faibles. Deux à trois ordres de grandeur sont ainsi gagnés sur la puissance de commande et sur la vitesse par rapport aux dispositifs analogues à onde de volume. On parle alors de quelques $\mu \mathrm{W} / \mathrm{MHz}$ pour des modulateurs de type interféromètre de Mach Zehnder. 

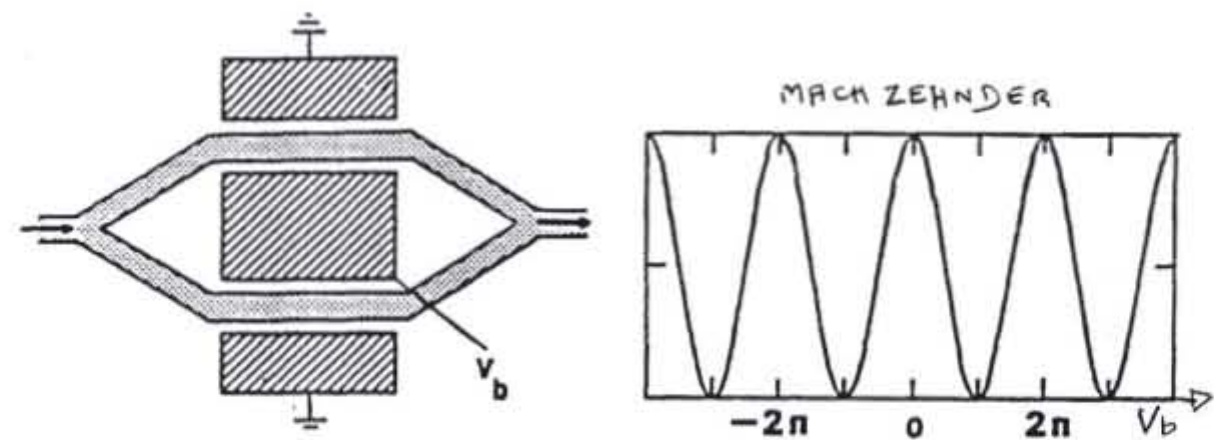

Il en est de même avec un dispositif sans équivalent en optique de volume et que seule l'optique guidée a permis de réaliser: le coupleur directif
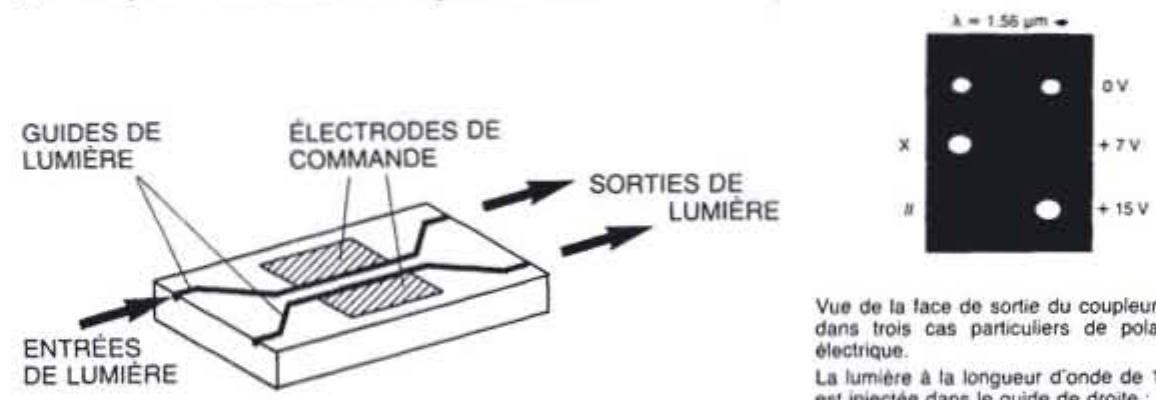

Vue de ta tace de sortie du coupleur directit Vue de la tace de sontie du coupleut directit
dans trois cas particuliers de polarisation electrique.

La lumiere a ta longueur donde de 1,56 $\mu \mathrm{m}$ est injectée dans le guide de droite

a) A tension nulle, la lumière sort des deux guides,

b) pour $7 \mathrm{~V}$, la lumiere sort presque entierement du guide couplé (etat X).

Schema de principe d'un coupleur directit optique a commande tlectrique : la lumiere c) pour $15 \mathrm{~V}$, la lumiere sort du guide direct peut sortir de run ou de rautre des deux guides suivant la tension electrique appliquete. (etat in)

. Ce composant a reçu une attention toute particulière du fait de sa capacité a accomplir diverses fonctions de première importance; il peut en effet servir de commutateur, de modulateur rapide, de multiplexeur temporel ou en longueur d'onde. Lui aussi tire avantageusement profit d'interactions électrooptiques Pockels transverses renforcées par l'effet de guidage monomode pour offrir des caractéristiques de modulation ou de commutation uniques: a 1,3 $\mu \mathrm{m}, 6 \mathrm{~V}$ de tension de commande, $<3 \mathrm{~dB}$ de perte d'insertion entre fibres monomodes et bande passante $>20 \mathrm{GHz}$. Le fonctionnement de ce composant est décrit par la théorie des modes couplés. 
Du fait de leurs propriétés radiatives, les matériaux semiconducteurs à bande interdite directe tels que GaAs et InP et leurs alliages ternaires ou quaternaires permettent de fabriquer des lasers extrêmement performants et compacts. La famille GaAs/GaAlAs est utilisé pour couvrir la fenêtre $0,8 \mu \mathrm{m}$, la famille InP/GaInAsP les fenêtres 1,3 et $1,5 \mu \mathrm{m}$. Un grand nombre de composants actifs et passifs a été fabriqué avec ces matériaux: laser et détecteur à fort rendement quantique, guide optique a faible perte $(<0,5 \mathrm{~dB} / \mathrm{cm})$, modulateur et commutateur électro-optiques à faible tension de commande $(<10 \mathrm{~V}$ ) et à grande rapidité ( $>20 \mathrm{GHz})$, bistable, filtre optique,...Ces matériaux ont en outre des qualités électriques telles, qu'ils présentent un grand intérêt en électronique rapide. Il est donc possible d'envisager l'association sur une même puce de tous les composants optiques et de l'électronique de commande du circuit. Ces puces portent le nom d'OEIC (OptoElectronic Integrated Circuit).

En dépit des problèmes technologiques multiples soulevés par cette intégration monolithique. les progrès sont notables: intégration de modules laser-détecteur d'asservissement ou lasermodulateur électro-absorbant fonctionnant à $>10 \mathrm{GHz}$ avec $<5 \mathrm{~V}$ ou encore lasercommutateur-amplificateur optique, tous ces prototypes de laboratoire étant capables d'émettre quelques $\mathrm{mW}$ vers $1,5 \mu \mathrm{m}$. La complexité de ces circuits est encore modeste et les performances souvent inférieures à celles que l'on pourrait avoir par hybridation, mais les perspectives qu'ils laissent déjà entrevoir en coût et performances sont très vastes.

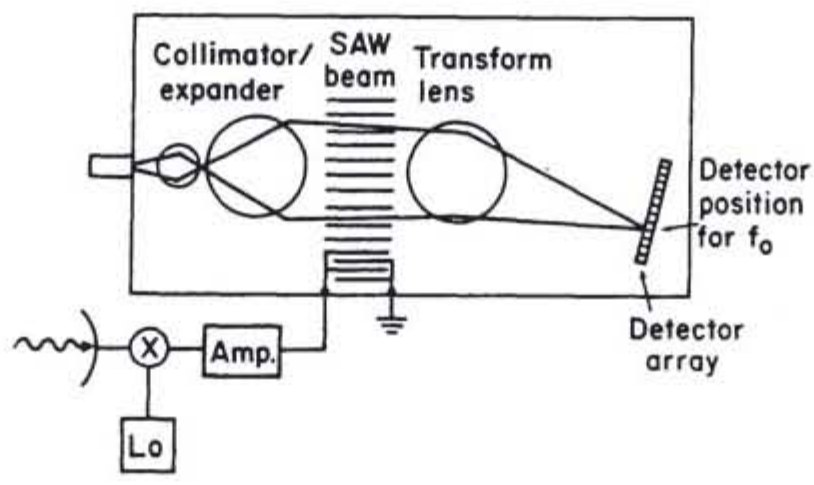

Diagram of an integrated-optic rf spectrum analyzer

C'est la raison pour laquelle ce domaine de l'optique qui permet d'exacerber les effets physiques et aussi d'en approcher de nouveaux est aujourd'hui en pleine effervescence. 


\section{5 - BIBLIOGRAPHIE}

* Ecole d'été d'Optoélectronique, Cargèse 1989, Les Editions de Physique

* R.G.HUNSPERGER : Integrated optics: Theory and Technology, Vol.33, Springer-Verlag

* A.K.GHATAK \& K.THYAGARAJAN, Optical Electronics, Cambridge University Press

* A.YARIV \& POCHI YEH : Optical waves in crystals, Wiley-interscience

* A.YARIV : Quantum electronics, J.Wiley Ed.

* J.SAPRIEL : Acousto-optique, Masson

* J.P.CASTERA, P.L.MEUNIER \& P.FRIEZ : Effets magnétooptiques dans les grenats et leur application à la réalisationd'un isolateur et d'un circulateur optiques, Revue Technique THOMSON-CSF, Vol.18, p255-299, Juin 1986

* J.SAULNIER : Cours d'optoélectronique, EFREI, Paris

* L.JEUNHOMME: Single-mode fiber optics, Optical Engineering/Vol.4, Dekker

\&\&\&\&\&\&\&\&\&\&\&\& 